\title{
MHD Nonlinear Mixed Convection Flow of Micropolar Nanofluid over Nonisothermal Sphere
}

\author{
Wubshet Ibrahim (iD) and Chaluma Zemedu \\ Department of Mathematics, Ambo University, Ambo, Ethiopia \\ Correspondence should be addressed to Wubshet Ibrahim; wubshetib@yahoo.com
}

Received 25 December 2019; Accepted 28 February 2020; Published 9 June 2020

Academic Editor: Mohamed Abd El Aziz

Copyright (C) 2020 Wubshet Ibrahim and Chaluma Zemedu. This is an open access article distributed under the Creative Commons Attribution License, which permits unrestricted use, distribution, and reproduction in any medium, provided the original work is properly cited.

\begin{abstract}
In this paper, two-dimensional steady laminar boundary layer flow of a nonlinear mixed convection flow of micropolar nanofluid with Soret and magnetic field effect over a nonisothermal sphere is evaluated. The mathematical formulation for the flow problem has been made with appropriate similarity transformation and dimensionless variables, and the main nonlinear boundary value problems were reduced into mixed high-order nonlinear ordinary differential equations. Solution for velocity, microrotation, temperature, and concentration has been obtained numerically. The equations were calculated using method bvp4c from Matlab software for various quantities of main parameters. The effects of various parameters on skin friction coefficient $f^{\prime \prime}(0)$, wall duo stress coefficient $-G^{\prime}(0)$, and convection mass transfer coefficient $-\Phi^{\prime}(0)$ are analysed and presented through the graphs and tables. The convergence test has been maintained. For the number of points greater than the suitable mesh number of points, the precision is not influenced but the set time is increased. Moreover, a comparison with a previous paper, obtainable in the literature, has been presented and an excellent agreement is obtained. The findings indicate that an increase in the values of nonisothermal parameters $(m, P)$, magnetic $\mathrm{Ma}$, thermal and solutal nonlinear convection $(\lambda, s)$ parameter, and Soret number is to enhance the temperature difference between the boundary layer and ambient fluid to diffuse which increases the velocity profile $f^{\prime}(\zeta)$ and their boundary layer thicknesses near the surface of the sphere.
\end{abstract}

\section{Introduction}

The investigation of MHD flow, heat and mass transfer has drawn attention of several researchers as a result of its request to numerous technological and different engineering and industrial developments, for instance, magnetic materials deal out, distillation of crude oil, magneto hydrodynamic electrical power creation, glass production, paper manufacturing, heat exchange devices, etc. Prasad et al. [1] have presented changeable thickness on mixed convection and heat transfer past an elastic vertical sheet with viscous electrically conducting fluid flow. However, the massive recirculation in reverse flow structure on combined convection past a heated sphere at small Reynolds number has been discussed by Kotouc et al. [2].

Subba et al. [3], Chamkha and Al-Mudhaf [4], and Balla and Naikoti [5] have reported the effects of convection slip on non-Newtonian nanofluid, magnetic field, and thermal radiation on a permeable sphere at uniform heat and mass fluxes with time dependent, correspondingly. The influences of changeable fluid density on the combined convection heat transfer have been analysed by Liping et al. [6]. Results show that the effect of changeable fluid density on velocity and temperature under gravity contains two branches: the free convection and forced convection parts. Gaffar et al. [7] have studied the behaviors of heat and mass on non-Newtonian Powell-Eyring fluid flow past an isothermal sphere. Findings indicate that temperature and concentration distributions exhibit increasing performance for enhance of the values of dimensionless stream-wise coordinate. Furthermore, the influence of changeable viscosity on variation of time in free convection MHD flow toward an isothermal sphere in a rotating system has been demonstrated by Dogbalous et al. [8]. 
Alzgool et al. [9] and Salleh and Nazar [10] have examined the mixed convection boundary layer flow starting from the solid sphere including thermal radiation effect and Newtonian heating in MHD flow micropolar fluid. Wubshet and Shanker [11] have reported the influences of magnetic field and thermal radiation on nanofluid past nonisothermal sphere along stretching sheet including nonisothermal surface temperature as well as nonisothermal heat flux. Recently, Wubshet and Chaluma [12] have investigated the impact of multiple slip conditions and thermal and solutal nonlinear convection on micropolar nanofluid flow over an isothermal sphere.

All the abovementioned studies have been on the flow over a plane, isothermal sphere, and in many other fields. Here, we examine numerically the nonlinear mixed convection flow of micropolar nanofluid over a nonisothermal sphere in the presence of magnetic and nonisothermal indexes and dimensionless stream-wise coordinate impacts, using bvp4c from Matlab. The impacts of physical parameters on fluid velocity, microrotation, temperature, and concentration are discussed and shown in graphs and tables

\section{Mathematical Formulation}

Consider a two-dimensional steady laminar boundary layer flow of a viscous micropolar nanofluid over a nonisothermal spherical body of unchanging radius $c$. Assume a nonisothermal surface temperature $T_{w}$ and the micropolar nanofluid is a constant concentration $C_{w}$. The temperature and concentration at far, correspondingly, are $T_{\infty}$ and $C_{\infty}$. The coordinates $x$ and $y$ are chosen such that $x$ measures the distance toward the surface of the sphere from the lower point and $y$ measures the distance normal to the surface of the sphere, as shown in Figure 1.

Following Amanulla et al. [13], the differential equations governing the present problem are given as follows:

$$
\begin{gathered}
\frac{\partial(u r)}{\partial x}+\frac{\partial(v r)}{\partial y}=0 \\
u \frac{\partial u}{\partial x}+v \frac{\partial u}{\partial y}=\left(v+\frac{\kappa}{\rho}\right) \frac{\partial^{2} u}{\partial y^{2}}+\frac{\kappa}{\rho} \frac{\partial H}{\partial y}+\left(g \left(R_{0}\left(T-T_{\infty}\right)\right.\right. \\
\left.+R_{t}\left(T-T_{\infty}\right)^{2}\right)+g\left(R_{0}\left(C-C_{\infty}\right)\right. \\
\left.\left.+R_{s}\left(C-C_{\infty}\right)^{2}\right)\right) \sin \left(\frac{x}{c}\right)+\sigma \frac{B_{0}^{2}}{\rho} u \\
u \frac{\partial H}{\partial x}+v \frac{\partial H}{\partial y}=\frac{\varphi}{\rho j} \frac{\partial^{2} H}{\partial y^{2}}-\frac{\kappa}{\rho j}\left(2 H+\frac{\partial u}{\partial y}\right) \\
u \frac{\partial T}{\partial x}+v \frac{\partial T}{\partial y}=\frac{\kappa}{\rho c_{p}} \frac{\partial^{2} T}{\partial y^{2}}+\gamma\left(D_{B} \frac{\partial C}{\partial y} \quad \frac{\partial T}{\partial y}\right)+\gamma D_{T} \frac{1}{T_{\infty}}\left(\frac{\partial T}{\partial y}\right)^{2} \\
u \frac{\partial C}{\partial x}+v \frac{\partial C}{\partial y}=\frac{D_{B} \partial^{2} C}{\partial y^{2}}+\frac{D_{c t} T_{k}}{T_{m}} \frac{\partial^{2} T}{\partial y^{2}}+\frac{D_{T}}{T_{\infty}} \frac{\partial^{2} T}{\partial y^{2}}
\end{gathered}
$$

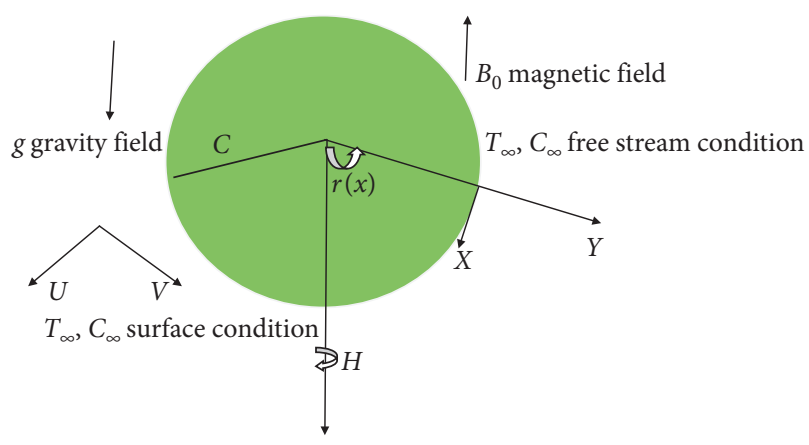

FIgURE 1: Flow diagram and coordinate system.

Subject to the proper velocity, microrotation, and concentration boundary conditions,

$$
\begin{aligned}
& u=a \frac{\partial u}{\partial y}, \\
& u=a \frac{\partial u}{\partial x}, \\
& v=0, \\
& H=-\delta \frac{\partial u}{\partial y}, \\
& H=-\delta \frac{\partial u}{\partial x}, \\
& C=C_{w}+e \frac{\partial C}{\partial y}, \\
& C=C_{w}+e \frac{\partial C}{\partial x}, \quad \text { at } y=0, \\
& u \longrightarrow u_{\infty}, \\
& H \longrightarrow 0, \\
& C \longrightarrow C_{\infty}, \quad \text { as } y \longrightarrow \infty .
\end{aligned}
$$

The proper thermal boundary conditions are

(1) The fixed surface temperature (FST):

$$
\begin{aligned}
& T=T_{w}=T_{\infty}+\frac{x^{m}}{c}, \\
& \frac{\partial T}{\partial x}=0, \quad \text { at } y=0, \\
& T \longrightarrow T_{\infty}, \quad \text { as } y \longrightarrow \infty .
\end{aligned}
$$


(2) The fixed heat flux (FHF):

$$
\begin{aligned}
-K \frac{\partial T}{\partial y} & =q_{w}=\frac{x^{P}}{c}, \\
-K \frac{\partial T}{\partial x} & =\frac{x^{P}}{c}, \quad \text { at } y=0, \\
T & \longrightarrow T_{\infty}, \quad \text { as } y \longrightarrow \infty,
\end{aligned}
$$

where $u$ and $v$ are the components of velocity in the $x$ and $y$ axes, correspondingly, $a$ stands for momentum slip factor, $e$ is solutal jump factor, $m$ and $P$ are integer constant, $H$ is the component of microrotation perpendicular to the $x y$ plane. Here, $\delta$ is a constant which lies between 0 and 1 stands for different categories of variations for concentration. When $\delta=0$, that is, the movement particle concentration is concentrated, then the microelements near to the wall surface are incapable to rotate; when $\delta=1 / 2$, the antisymmetric part of the stress tensor disappears, which stands for puny concentration. The case $\delta=1$ is meant for modeling the turbulent boundary layer flows. $\mu$ stands for the coefficient of fluid viscosity, $\rho$ is the density, $\nu=\mu / \rho$ is kinematic viscosity, $c_{p}$ stands for the specific heat, $j=d^{2} / \sqrt{\mathrm{Gr}}$ is the microinertia per unit mass, $\varphi=(\mu+$ $(\kappa / 2)) j$ is spin gradient viscosity, $\kappa$ is the vortex viscosity, $T$ stands for temperature, $\mathrm{K}$ is the thermal conductivity of the fluid, $T_{w}=\left(T_{\infty}+\Delta T\right)$ and $C_{w}=\left(C_{\infty}+\Delta C\right)$ represent the variables temperature and concentration at surface, where $\Delta T$ and $\Delta C$ are constants which give the rate of growth of temperature and concentration alongside the surface and $T_{\infty}$ and $C_{\infty}$ stand for the uniform temperature, concentration of the free stream, and $g$ represents gravity. $\nabla \rho / \rho=\left(R_{0}\left(T-T_{\infty}\right)\right.$ $\left.+R_{t}\left(T-T_{\infty}\right)^{2}+R_{0}^{*}\left(C-C_{\infty}\right)+R_{s}\left(C-C_{\infty}\right)^{2}\right)$, where $R_{0}$ and $R_{0}^{*}$ are constants and $R_{t}$ and $R_{s}$ are the constant coefficients of thermal and volumetric expansion, respectively. This relation will be nonlinear density temperature and concentration (NDTC) variation. $\gamma=(\rho c)_{p} /(\rho c)_{f}$ is the ratio between the effective heat capacity of the nanoparticle material and the heat capacity of the fluid, $D_{\text {ct }}$ represents the Soret-type diffusivity, $T_{m}$ is fluid mean temperature, $T_{k}$ is the thermal diffusion ratio, $D_{T}$ stands for the thermophoretic diffusion coefficient, $\sigma$ is electrical conductivity, and $B_{0}$ represents magnetic field. Let $r(x)=c \quad \sin (x / c)$ be the radial distance from the symmetrical axis to the surface of the sphere.
Following Amanulla et al. [13], the non dimensional variables are as follows:

$$
\begin{aligned}
\varepsilon & =\frac{x}{c}, \\
\zeta & =\frac{(\mathrm{Gr})^{1 / 4}}{c} y, \\
r & =\frac{r^{*}}{c}, \\
f & =\frac{\psi}{\nu \mathcal{E}(\mathrm{Gr})^{1 / 4}}, \\
G & =\frac{c^{2}}{\nu \mathcal{E}(\mathrm{Gr})^{3 / 4}} H, \\
\Phi & =\frac{C-C_{\infty}}{C_{w}-C_{\infty}} .
\end{aligned}
$$

For thermal boundary conditions, we consider the nondimensional quantities as follows:

(a) For fixed surface temperature, $m$ is the wall temperature parameter, $T_{w}$ is the temperature at the wall, and $c$ is the constant. The dimensionless temperature in FST can be selected as

$$
\vartheta=\frac{T-T_{\infty}}{T_{w}-T_{\infty}} .
$$

(b) For fixed heat flux FHF, the wall heat flux is taken to be changeable with radial distance $r(x)$ from the symmetrical axis to the surface of the sphere, and we introduce the dimensionless temperature as

$$
\theta=\frac{T-T_{\infty}}{T_{w}-T_{\infty}},
$$

where $T_{w}-T_{\infty}=\left(x^{P} / c\right)\left(c / \mathrm{Gr}^{1 / 4}\right)$.

The continuity equation can be integrated by introducing a stream function $\psi(x, y)$ as $u r=\partial r \quad \psi / \partial y$ and $v r=-(\partial r \quad \psi / \partial x)$, which satisfies the continuity equation (1). Hence, equations (2)-(5) can be written in the following nonlinear system of PDEs:

(a) For FST case,

$$
\begin{gathered}
(1+\alpha) f^{\prime \prime \prime}+\left((1+\varepsilon \quad \cot (\varepsilon)) f+\varepsilon \frac{\partial f}{\partial \varepsilon}\right) f^{\prime \prime}-\varepsilon \frac{\partial f^{\prime}}{\partial \varepsilon} f^{\prime}-\left(f^{\prime}\right)^{2}+M a f^{\prime}+\alpha G^{\prime}+\left(\vartheta+\lambda \vartheta^{2}\right) \frac{\sin (\varepsilon)}{\varepsilon}+\frac{G m}{G r}\left(\Phi+s \Phi^{2}\right) \frac{\sin (\varepsilon)}{\varepsilon}=0 \\
\left(1+\frac{\alpha}{2}\right) G^{\prime \prime}+(1+\varepsilon \cot (\varepsilon)) f G^{\prime}-G f^{\prime}-\alpha\left(2 G+f^{\prime \prime}\right)+\varepsilon\left(\frac{\partial f}{\partial \varepsilon} G^{\prime}-\frac{\partial G}{\partial \varepsilon} f^{\prime}\right)=0 \\
\frac{1}{\operatorname{Pr}} g^{\prime \prime}+(1+\varepsilon \cot (\varepsilon)) f \vartheta^{\prime}+\varepsilon\left(\frac{\partial f}{\partial \varepsilon} g^{\prime}-\frac{\partial \vartheta}{\partial \varepsilon} f^{\prime}\right)+N t\left(\vartheta^{\prime}\right)^{2}-m \vartheta f^{\prime}+N b \Phi^{\prime} \vartheta^{\prime}=0 \\
\frac{1}{\operatorname{PrLe}} \Phi^{\prime \prime}+\operatorname{So} \vartheta^{\prime \prime}+\frac{N t}{\operatorname{NbPrLe}} \vartheta^{\prime \prime}+\left((1+\varepsilon \cot (\varepsilon)) f+\varepsilon \frac{\partial f}{\partial \varepsilon}\right) \Phi^{\prime}-\varepsilon \frac{\partial \Phi}{\partial \varepsilon} f^{\prime}=0
\end{gathered}
$$


Subject to the boundary conditions,

$$
\begin{aligned}
\text { at } \zeta & =0: f^{\prime}(0)=b v f^{\prime \prime}(0), \\
\frac{\partial f(0)}{\partial \varepsilon} & =0, \\
\vartheta(0) & =1, \\
\frac{\partial \vartheta(0)}{\partial \varepsilon} & =0, \\
G(0) & =-\delta f^{\prime \prime}(0), \\
\frac{\partial G(0)}{\partial \varepsilon} & =b h f^{\prime \prime}(0), \\
\Phi(0) & =1+b m \Phi^{\prime}(0), \\
\frac{\partial \Phi(0)}{\partial \varepsilon} & =G r^{1 / 4} \Phi^{\prime}(0), \\
\text { as } \zeta \longrightarrow f^{\prime} & =0, \\
\vartheta & =0 \\
G & =0, \\
\Phi & =0 .
\end{aligned}
$$

(b) For FHF case,

$$
\begin{aligned}
& \frac{1}{\operatorname{Pr}} \theta^{\prime \prime}+(1+\varepsilon \quad \cot (\varepsilon)) f \theta^{\prime}+\varepsilon\left(\frac{\partial f}{\partial \varepsilon} \theta^{\prime}-\frac{\partial \theta}{\partial \varepsilon} f^{\prime}\right)+\operatorname{Nt}\left(\theta^{\prime}\right)^{2} \\
& -P \theta f^{\prime}+\operatorname{Nb}^{\prime} \theta^{\prime}=0 .
\end{aligned}
$$

Subject to the boundary conditions,

$$
\begin{aligned}
\frac{\partial f(0)}{\partial \varepsilon} & =0, \\
\theta^{\prime}(0) & =-1, \\
\frac{\partial \theta(0)}{\partial \varepsilon} & =-1-\frac{P \theta}{\varepsilon}, \\
G(0) & =-\delta f^{\prime \prime}(0), \\
\frac{\partial G(0)}{\partial \varepsilon} & =b h f^{\prime \prime}(0), \\
\Phi(0) & =1+b m \Phi^{\prime}(0), \\
\frac{\partial \Phi(0)}{\partial \varepsilon} & =G r^{1 / 4} \Phi^{\prime}(0), \\
\text { as } \zeta \longrightarrow f^{\prime} & =0, \\
\theta & =0, \\
G & =0, \\
\Phi & =0,
\end{aligned}
$$$$
\text { at } \zeta=0: f^{\prime}(0)=b v f^{\prime \prime}(0) \text {, }
$$

where prime represent differential with respect to $\zeta$, $\varepsilon=x / c$ stands for dimensionless stream-wise coordinate, $\mathrm{bv}=a \mathrm{Gr}^{1 / 4} / c$ is velocity slip parameter, $\mathrm{bh}=$ $\delta c+\delta^{2} / \varepsilon$ denotes microrotation slip parameter, $\mathrm{bm}=e \mathrm{Gr}^{1 / 4} / c$ stands for solutal jump parameter, $\alpha=$ $\kappa / \mu$ the is material parameter, $\mathrm{Gr}=c^{3} g R_{0}$ $\left(T_{w}-T_{\infty}\right) / \nu^{2}$ represents the ratio of the buoyancy force which arise from temperature difference to the viscous force times inertia force to viscous force termed as thermal Grashof number, $\mathrm{Gm}=c^{3} g R_{0}^{*}$ $\left(C_{w}-C_{\infty}\right) / \nu^{2}$ represents the ratio of the buoyancy force which arise from concentration difference to the viscous force times inertia force to viscous force termed as solutal Grashof number, and $\lambda=\left(R_{t} / R_{0}\right)\left(T_{w}-T_{\infty}\right)$ and $s=\left(R_{s} / R_{0}^{*}\right)\left(C_{w}-C_{\infty}\right)$ are thermal and solutal nonlinear convection parameters, respectively. We observe that, for $\lambda=s=0$, the flow of equation (12) becomes a convective micropolar nanofluid. Also, the relation of buoyancy force $(\mathrm{Gm}: \mathrm{Gr})$ can be seen in the equation is the dimensionless parameter indicating the ratio between the buoyancy force caused by concentration change and the buoyancy force owing to temperature change. Furthermore, Gm $=0$ is for nonbuoyancy influence because of mass diffusion, $\mathrm{Gr} \longrightarrow \infty$ i.e., $\mathrm{Gm} / \mathrm{Gr} \approx 0$ is for nonbuoyancy effect owing to thermal diffusion and $\mathrm{Gm} / \mathrm{Gr}=1$ is for thermal and mass buoyancy forces of the same strength. So = $\left(D_{\mathrm{ct}} T_{k}\left(T_{w}-T_{\infty}\right) / T_{m} \nu\left(C_{w}-C_{\infty}\right)\right)$ stands for Soret number, $\operatorname{Pr}=\left(\rho \nu c_{p} / K\right)$ is Prandtl number, Le $=K / D_{B} \rho c_{p}$ is Lewis number, $\mathrm{Nb}=\left((\rho c)_{p} /\right.$ $\left.(\rho c)_{f}\right)\left(D_{B} / \nu\right)\left(C_{w}-C_{\infty}\right)$ stands for Brownian motion parameter, $\mathrm{Nt}=\left((\rho c)_{p} /(\rho c)_{f}\right)\left(D_{T} / T_{\infty} \nu\right)\left(T_{w}-\right.$ $\left.T_{\infty}\right)$ represents thermophoresis parameter, $\mathrm{Ma}=(\sigma / \rho) B_{0}^{2}\left(c^{2} / \nu G r^{1 / 2}\right)$ is magnetic parameter, and $m$ stands for power index of terms in the nonisothermal surface.

It can be appeared that, in the lower point of the sphere $\varepsilon \approx 0$, equations (12)-(18) reduced to the next nonlinear system of ordinary differential equations.

(a) For FST case,

$$
\begin{gathered}
(1+\alpha) f^{\prime \prime \prime}+f f^{\prime \prime}-\left(f^{\prime}\right)^{2}+M a f^{\prime}+\alpha G^{\prime}+\left(\vartheta+\lambda \vartheta^{2}\right) \\
+\frac{\mathrm{Gm}}{\mathrm{Gr}}\left(\Phi+s \Phi^{2}\right)=0, \\
\left(1+\frac{\alpha}{2}\right) G^{\prime \prime}+f G^{\prime}-G f^{\prime}-\alpha\left(2 G+f^{\prime \prime}\right)=0, \\
\frac{1}{\operatorname{Pr}} \vartheta^{\prime \prime}+f \vartheta^{\prime}+\mathrm{Nt}\left(\vartheta^{\prime}\right)^{2}-m \vartheta f^{\prime}+\mathrm{Nb} \Phi^{\prime} \vartheta^{\prime}=0, \\
\frac{1}{\operatorname{PrLe}} \Phi^{\prime \prime}+\operatorname{So} \vartheta^{\prime \prime}+\frac{\mathrm{Nt}}{\mathrm{NbPrLe}} \vartheta^{\prime \prime}+f \Phi^{\prime}=0 .
\end{gathered}
$$


(b) For FHF case,

$$
\frac{1}{\operatorname{Pr}} \theta^{\prime \prime}+f \theta^{\prime}+\mathrm{Nt}\left(\theta^{\prime}\right)^{2}-P \theta f^{\prime}+\mathrm{Nb} \Phi^{\prime} \theta^{\prime}=0 \text {. }
$$

Subject to the boundary conditions,

$$
\begin{aligned}
\text { at } \zeta & =0: f^{\prime}(0)=b v f^{\prime \prime}(0), \\
\vartheta(0) & =1, \\
G(0) & =-\delta f^{\prime \prime}(0), \\
\Phi(0) & =1+b m \Phi^{\prime}(0), \\
\text { as } \zeta \longrightarrow \infty: f^{\prime} & =0 \\
\vartheta & =0 \\
G & =0 \\
\Phi & =0 .
\end{aligned}
$$

The physical quantities of awareness in this problem are the limited skin friction coefficient $C_{f}$, wall couple stress $m_{w}$, the Nusselt number $\mathrm{Nu}$, and the Sherwood Sh number, and they can be written as follows:

$$
\begin{aligned}
C_{f} & =\frac{\tau_{w}}{\rho\left(u_{w}\right)}, \\
\mathrm{Nu} & =\frac{q_{w}}{\kappa\left(T_{w}-T_{\infty}\right)}, \\
\mathrm{Sh} & =\frac{q_{m}}{D_{B}\left(C_{w}-C_{\infty}\right)}, \\
m_{w} & =-\left(\nu+\frac{\kappa}{2 \rho}\right) j\left(\frac{\partial H}{\partial y}\right)_{(y=0)},
\end{aligned}
$$

where $\quad \tau_{w}=(\mu+\kappa)(\partial u / \partial y)_{(y=0)}+\kappa(H)_{0}$, $q_{w}=-K((\partial T / \partial y))_{(y=0)}$, and $q_{m}=-D_{B}(\partial C / \partial y)_{(y=0)}$.

Using the nondimensional variables (9) and (10) and boundary condition (18), the limited skin friction coefficient $C_{f}$, wall couple stress $m_{w}$, the Nusselt number $\mathrm{Nu}$, and the Sherwood number Sh are

$$
\begin{aligned}
f^{\prime \prime}(0) & =\frac{C_{f} a \varepsilon \mathrm{Gr}^{3 / 4}}{c^{2}(1+\alpha(1-\delta))}, \\
-G^{\prime}(0) & =\frac{m_{w} c}{v^{2} \varepsilon a(1+(\alpha / 2)) \sqrt{\mathrm{Gr}}}, \\
\mathrm{Nu} \frac{c}{\mathrm{Gr}^{1 / 4}} & =-\vartheta^{\prime}(0), \\
\operatorname{Sh} \frac{c}{\mathrm{Gm}^{1 / 4}} & =-\Phi^{\prime}(0) .
\end{aligned}
$$

\section{Numerical Solution}

The five-pair high-order ordinary differential equations (19)-(23), subjected to the boundary condition equation (18), are examined numerically using the function bvp4c from Matlab software for different values of physical parameters, that is, slip parameters (bv, bh, bm), Prandtl number Pr, thermal Grashof number Gr, Solutal Grashof number $\mathrm{Gm}$, Brownian motion parameter $\mathrm{Nb}$, thermophoresis parameter $\mathrm{Nt}$, Lewis number Le, thermal nonlinear convection parameter $\lambda$, solutal nonlinear convection parameter $s$, material parameter $\alpha$, microrotation parameter $\delta$, dimensionless stream-wise coordinate $\varepsilon$, Magnetic parameter $\mathrm{Ma}$, and nonisothermal parameters $(m, P)$.

Mathematical results are found using Matlab BVP solver bvp4c from Matlab which is a finite difference code that make the three-stage Lobatto IIIa formulation real. To apply bvp4c from Matlab, first, equations (12)-(18) are changed into a system of first-order equations.

Second, establish the boundary value problem and use bvp solver bvp4c from Matlab. In solving the BVP by means of Matlab, bvp4c has only three points of views: a function ODEs for calculation of the residual in the boundary conditions, and a building solint that gives a guess for a mesh. The ODEs are handled exactly as in Matlab IVP solvers. Further clarification on the procedure of bvp4c is found in the book by Shampine et al. [14].

Let $\quad y(1)=f, \quad y(2)=f^{\prime}, \quad y(3)=f^{\prime \prime}, \quad y(4)=h$, $y(5)=h^{\prime}, \quad y(6)=\vartheta, \quad y(7)=9^{\prime}, \quad y(8)=\Phi, \quad y(9)=\Phi^{\prime}$, $y(10)=\partial f / \partial \varepsilon, \quad y(11)=\partial h / \partial \varepsilon, \quad y(12)=\partial 9 / \partial \varepsilon, \quad y(13)=\partial$ $\Phi / \partial \epsilon$, and $y(14)=\partial f^{\prime} / \partial \epsilon$, and $y=\left[f, f^{\prime}, f^{\prime \prime}, h, h^{\prime}, \vartheta, \vartheta^{\prime}, \Phi\right.$, $\left.\Phi^{\prime}, \partial f / \partial \varepsilon, \partial h / \partial \varepsilon, \partial \vartheta / \partial \varepsilon, \partial \Phi / \partial \varepsilon, \partial f^{\prime} / \partial \varepsilon\right]^{T}$ gives 


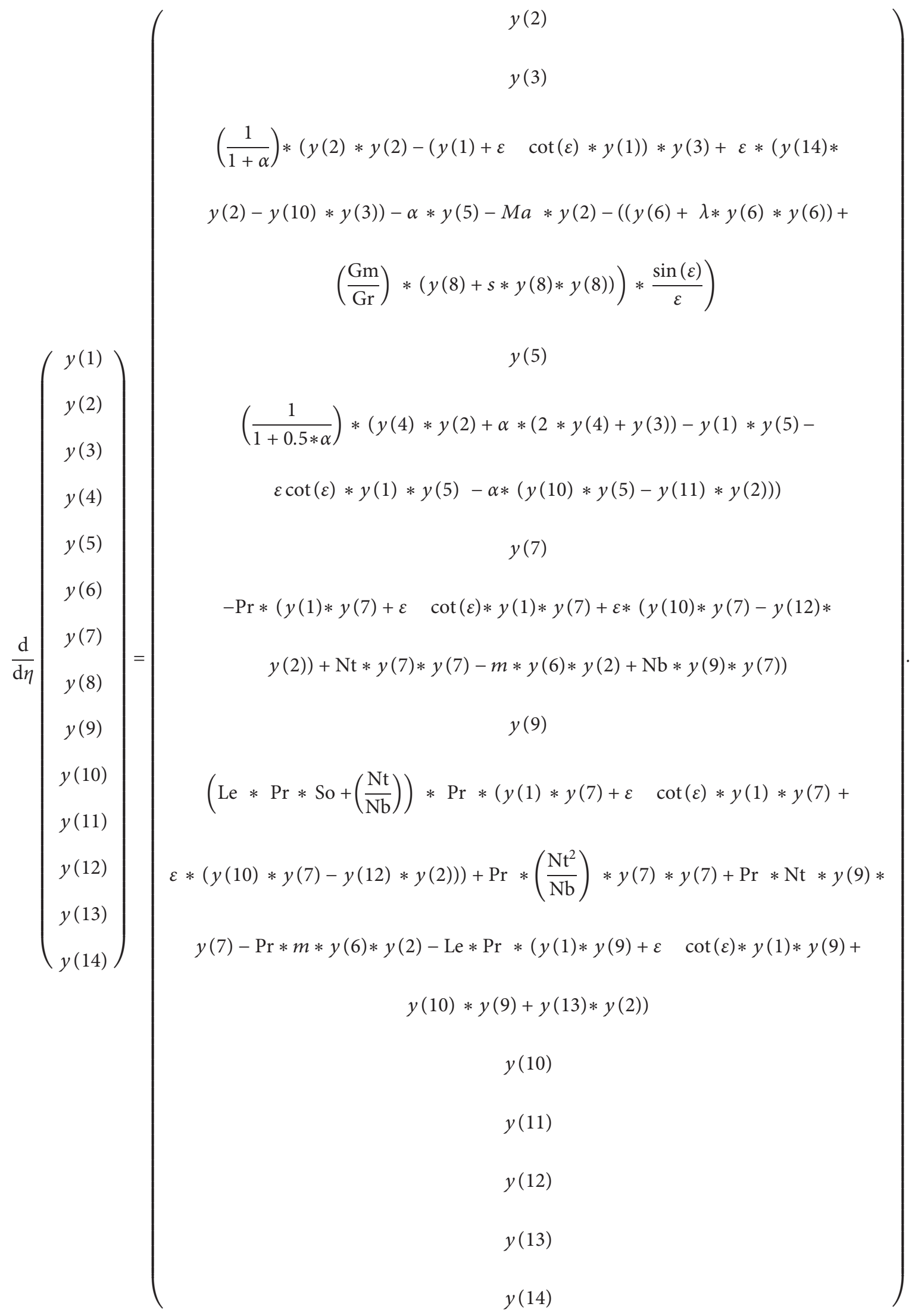




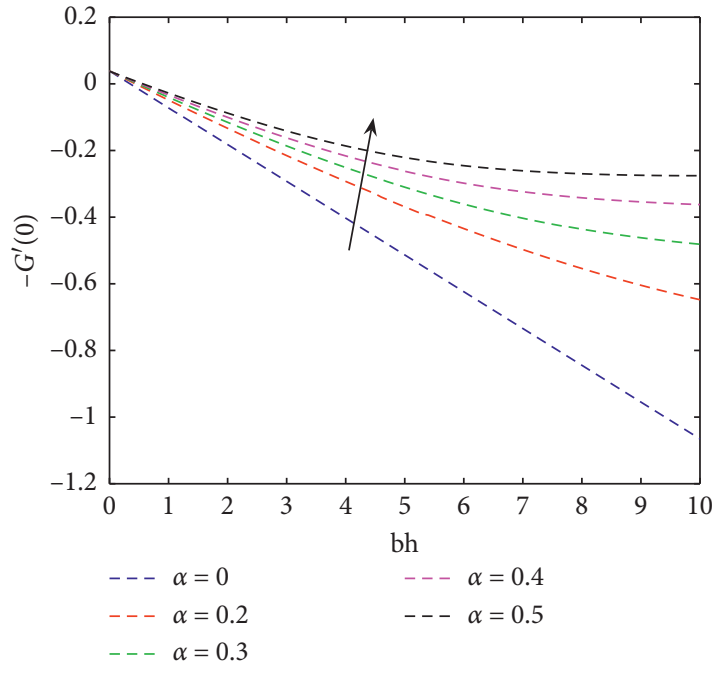

Figure 2: $-\mathrm{G}^{\prime}(0)$ profile for different values of $\alpha$ when $\mathrm{Gr}=1.5$, $\mathrm{Gm}=10, \quad \mathrm{Nt}=0.7, \quad m=1, \quad \varepsilon=20, \quad \alpha=s=\mathrm{Ma}=0.1$, $\mathrm{bm}=\mathrm{bv}=\mathrm{Le}=n=\lambda=\mathrm{Nb}=\mathrm{So}=0.5$, and $\mathrm{Pr}=0.72$.

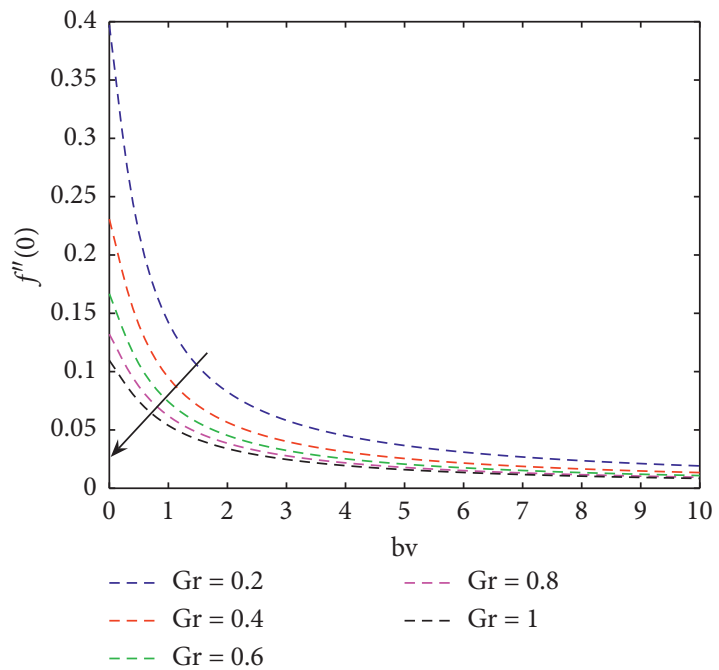

Figure 3: $f^{\prime}(0)$ graph for different values of $\mathrm{Gr}$ when $\mathrm{Gm}=6.5$ and $\mathrm{Nt}=m=1$.

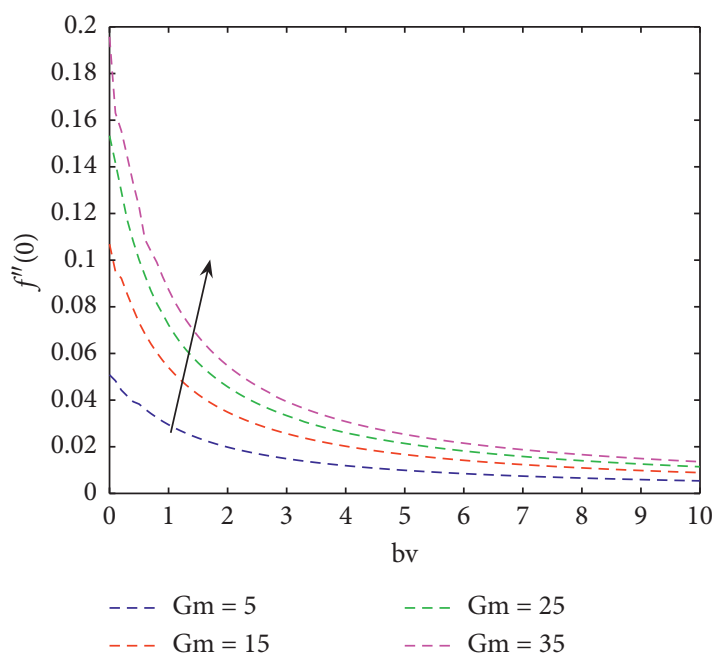

Figure 4: $f^{\prime \prime}$ profile for different values of $\mathrm{Gm}$ when $\mathrm{Gr}=2$ and $\mathrm{Nt}=m=1$.

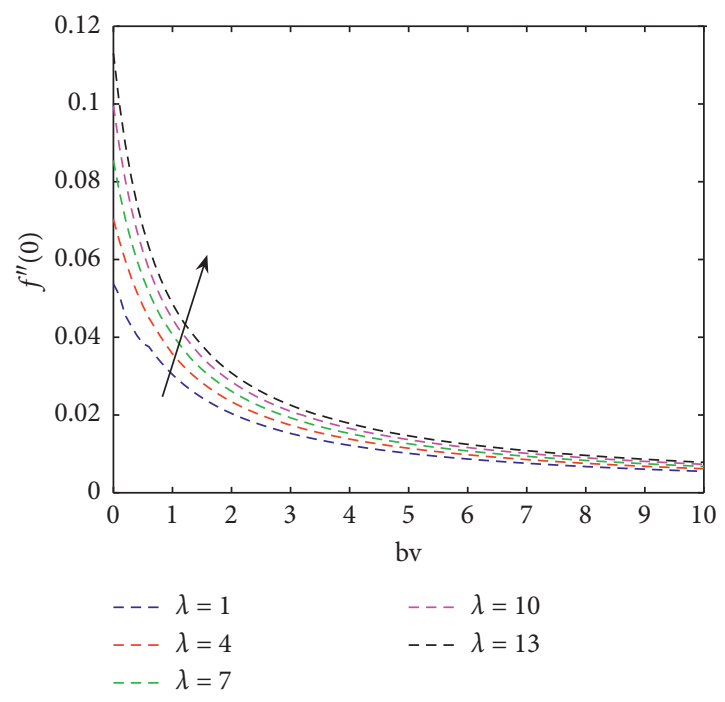

FIGURE 5: $f^{\prime \prime}(0)$ graph for different values of $\lambda$ when $\mathrm{Gr}=2, \mathrm{Gm}=5$, and $\mathrm{Nt}=m=1$.

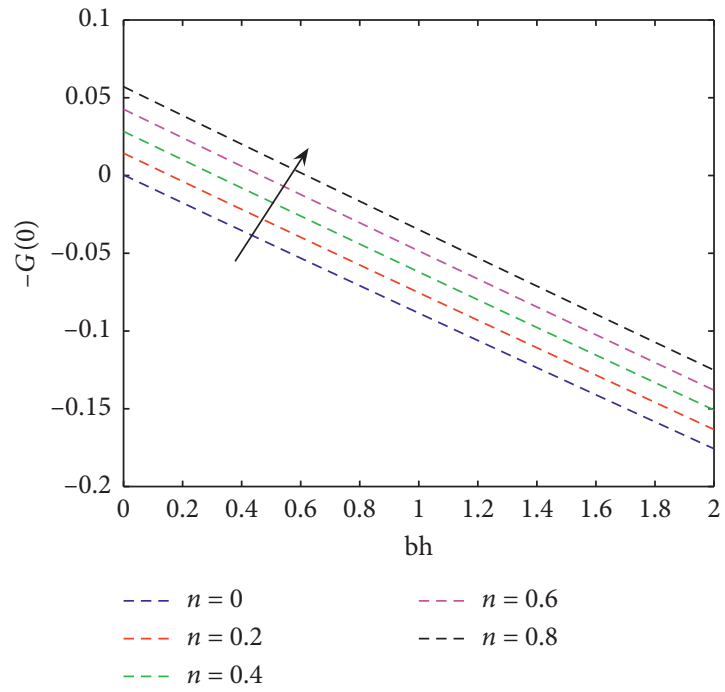

Figure 6: $G^{\prime}(0)$ profile for different values of $\mathrm{n}$ when $\mathrm{Gr}=1.5$, $m=1, \mathrm{Gm}=9$, and $\mathrm{Nt}=0.7$.

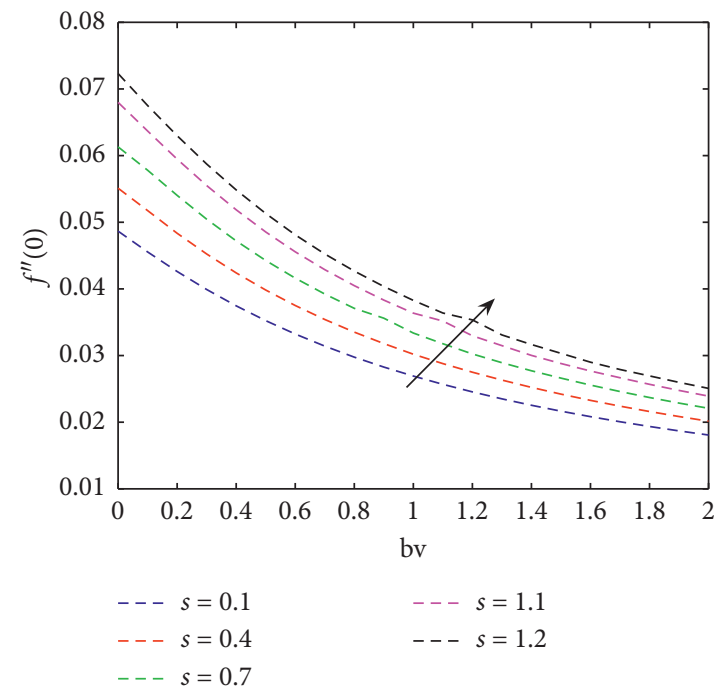

Figure 7: $f^{\prime \prime}$ profile for different values of $s$ when $\mathrm{Gr}=2, \mathrm{Gm}=5$, and $\mathrm{Nt}=m=1$. 


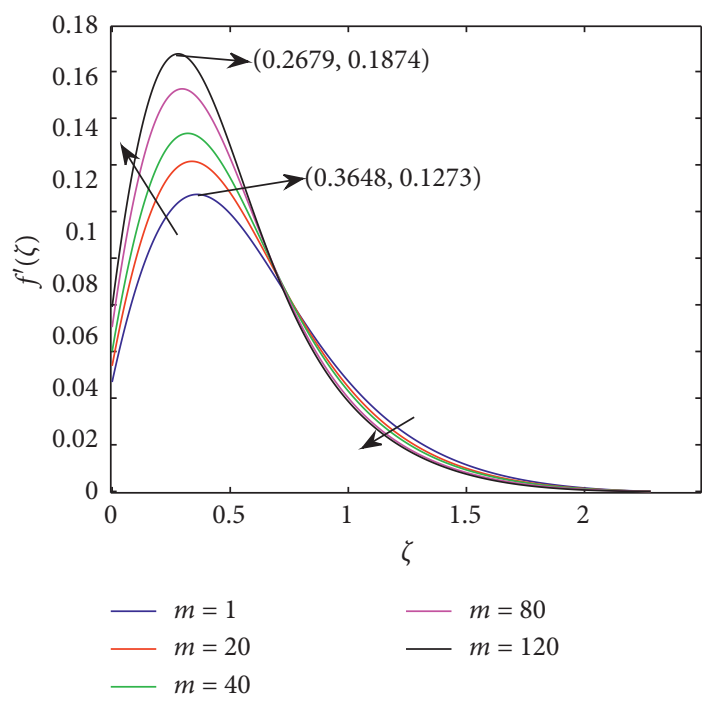

Figure 8: $f^{\prime}$ profile for different values of $\mathrm{m}$ when $\mathrm{Gr}=\mathrm{bv}=0.1, \mathrm{Gm}=10, s=0.24, \mathrm{Ma}=0.11, \mathrm{Le}=0.54$, and $\mathrm{Nt}=0.1$.

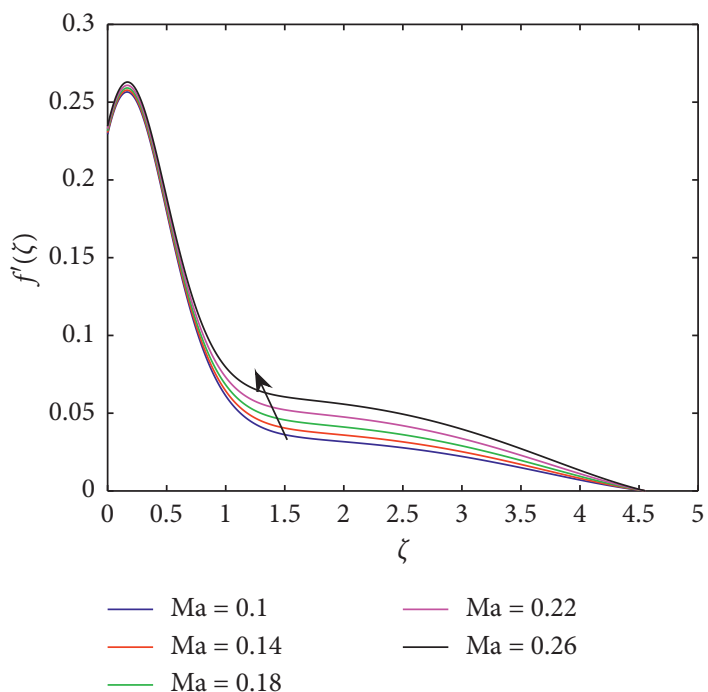

Figure 9: $f^{\prime}(\zeta)$ graph for different values of Ma when $\varepsilon=0.2, \mathrm{Gr}=0.1, m=1, s=0.27, \mathrm{Nt}=0.4, \operatorname{Pr}=\alpha=0.72\left(\left(-b \pm \sqrt{b^{2}-4 a c}\right) / 2 a\right)$.

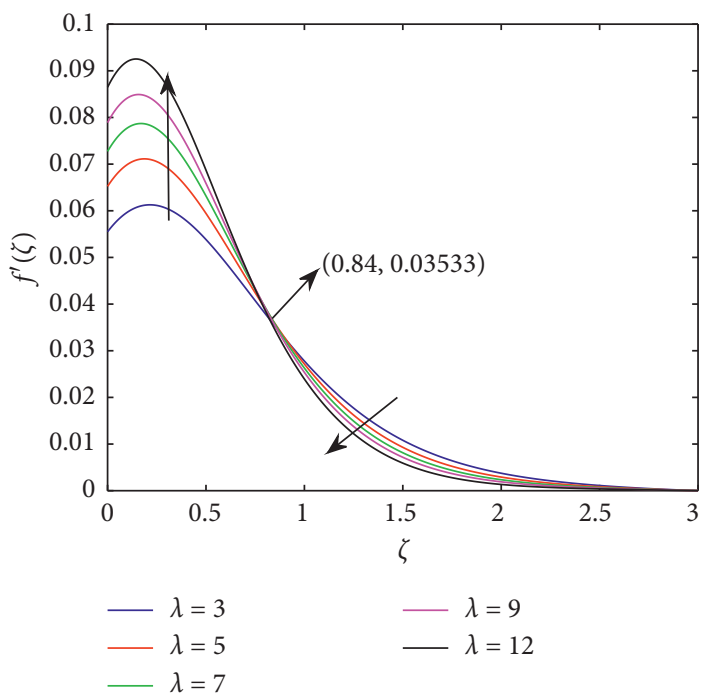

Figure 10: $f^{\prime}$ profile for different values of $\lambda$ when $P=1$ and $\mathrm{Nt}=\mathrm{Gr}=\mathrm{Gm}=0.1$. 


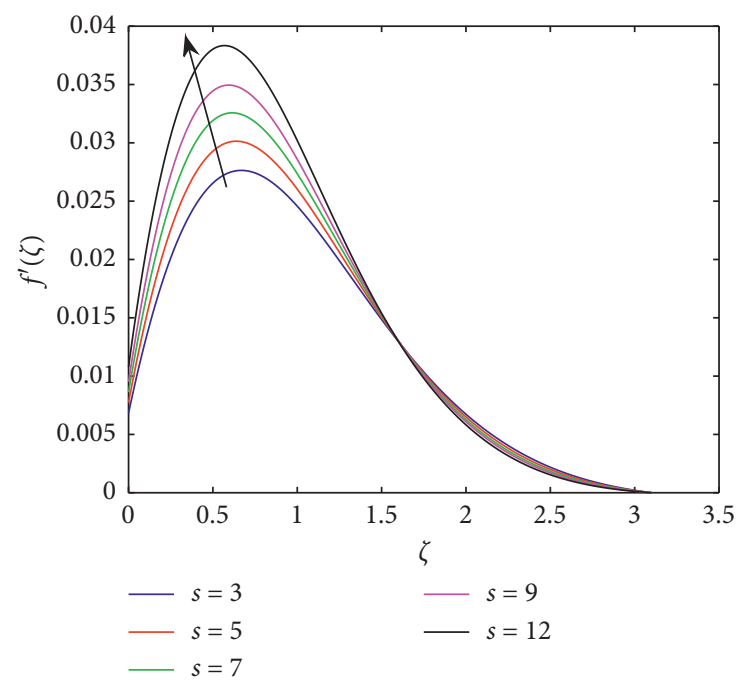

FIgURE 11: $f^{\prime}(\zeta)$ graph for different values of $\mathrm{s}$ when $\mathrm{PP}=\mathrm{Gr}=\mathrm{Gm}=1$.

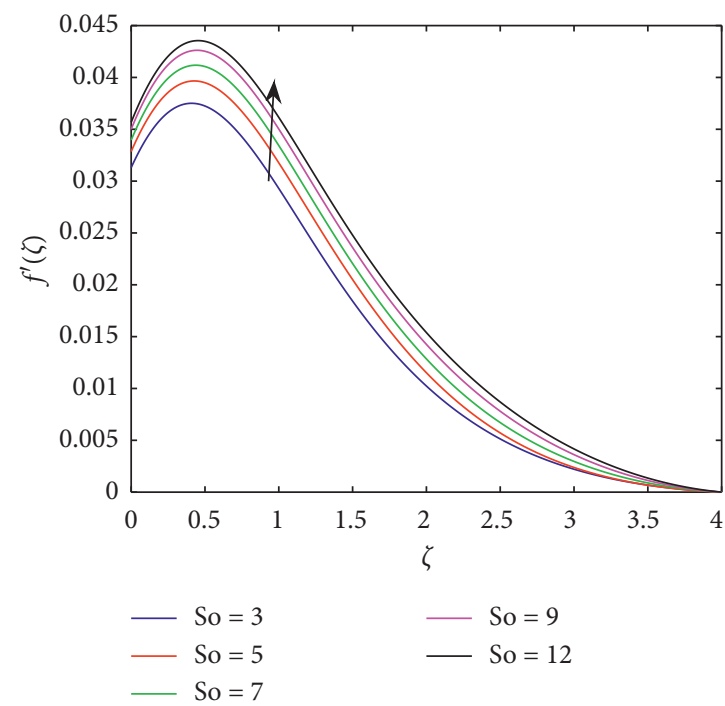

Figure 12: $f^{\prime}(\zeta)$ profile for different values of So when $P=\mathrm{Gr}=\mathrm{Gm}=\mathrm{bv}=1$ and $\mathrm{Nt}=0.1$.

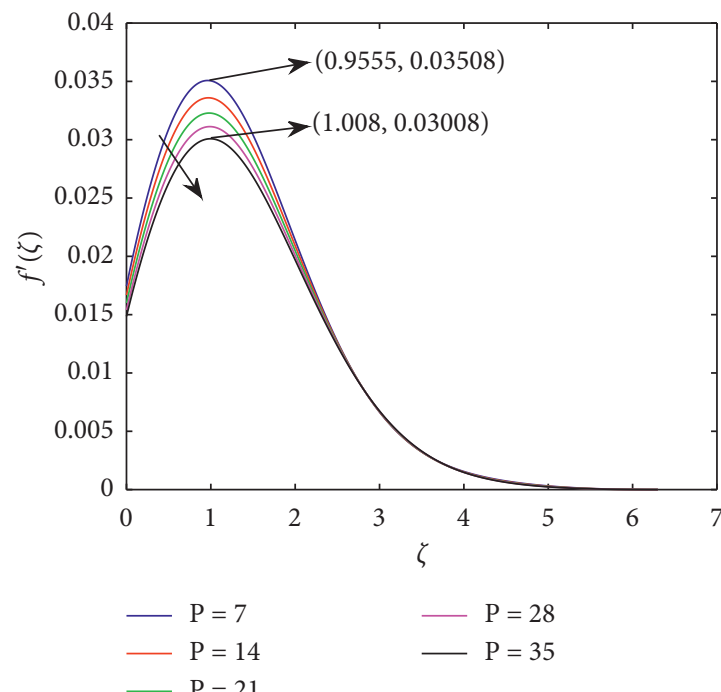

Figure 13: $f^{\prime}(\zeta)$ graph for different values of $P$ when $\mathrm{Gr}=1.2, \mathrm{Gm}=2.1, \mathrm{bv}=0.5, s=0.72, \mathrm{Nt}=0.5, \alpha=5, \mathrm{Nb}=\mathrm{bm}=\mathrm{bh}=0.1$, and $\lambda=0.7$. 


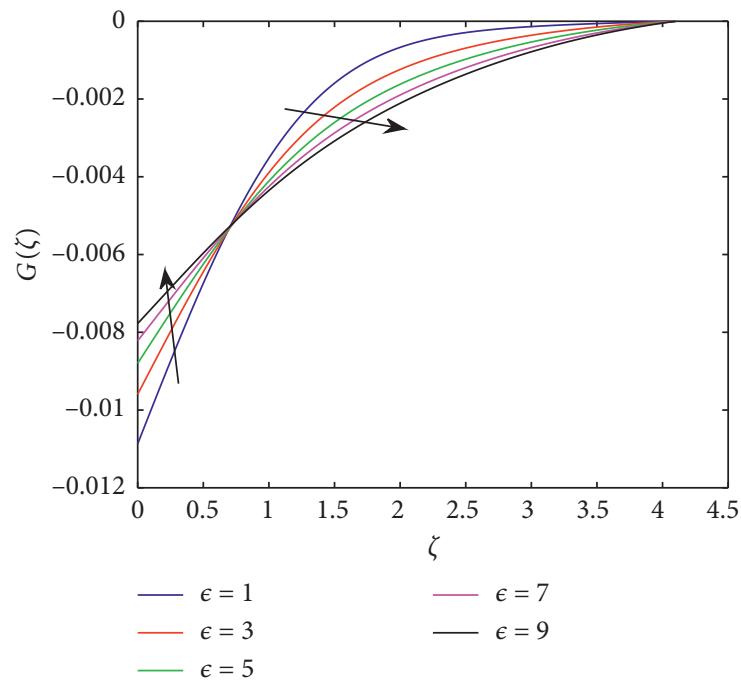

FIgURE 14: $G(\zeta)$ profile for different values of $\varepsilon$ when $\mathrm{Gm}=20, m=1, s=0.1, \mathrm{Nb}=1, \lambda=0.5, \mathrm{Gr}=18$, and $\mathrm{Nt}=0.1$.

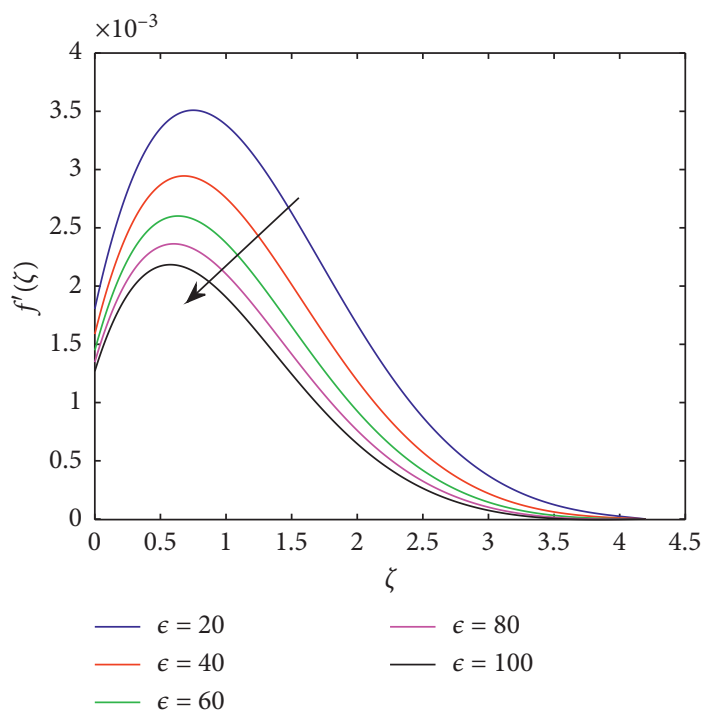

Figure 15: $f^{\prime}(\zeta)$ graph for different values of $\mathcal{\varepsilon}$ when $\mathrm{Gr}=13, \mathrm{Gm}=m=1$, bv $=0.35, \mathrm{Nb}=0.1$, and $\mathrm{Nt}=0.28$.

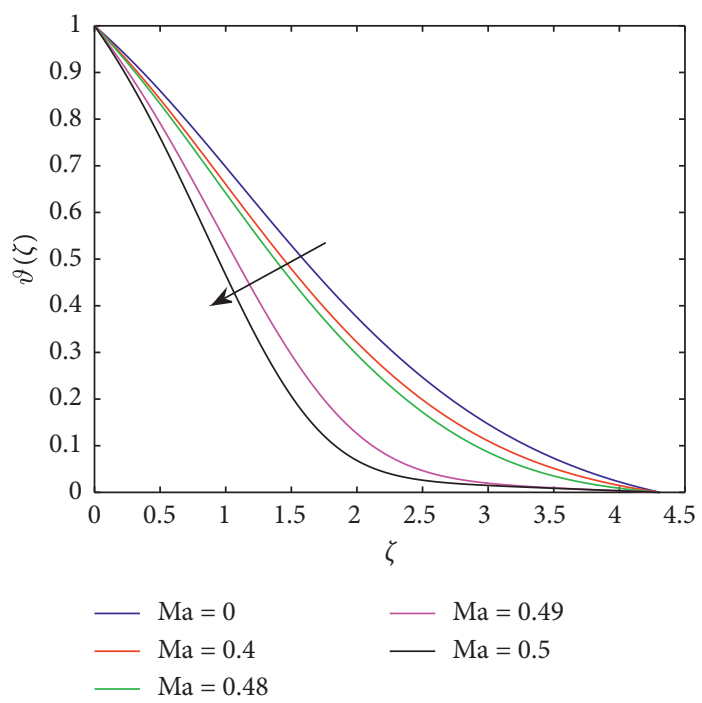

Figure 16: $\vartheta(\zeta)$ profile for different values of Ma when $\mathrm{Gr}=1.25, \mathrm{Gm}=s=0.1, \alpha=0.3, \lambda=\mathrm{bv}=\mathrm{bm}=\mathrm{bh}=\mathrm{Le}=\mathrm{Nb}=\mathrm{So}=n=0.5, m=1$, $\mathrm{Nt}=3, \operatorname{Pr}=0.77$, and $\varepsilon=20$, for FST. 


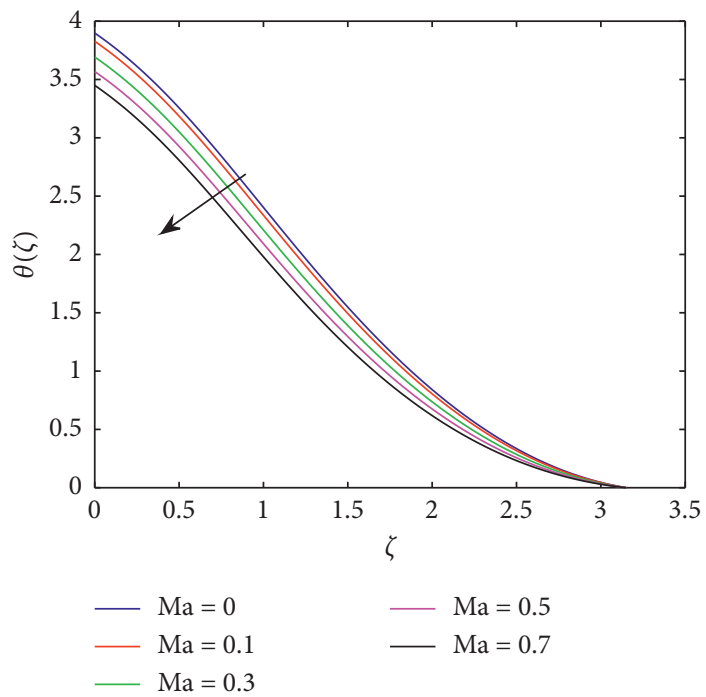

Figure 17: $\theta(\zeta)$ profile for different values of Ma when $\mathrm{Gm}=\mathrm{Gr}=\mathrm{bm}=s=\mathrm{bh}=\alpha=\lambda=\mathrm{Nb}=0.1, \mathrm{bv}=\mathrm{Le}=\mathrm{So}=n=0.5, P=1, \mathrm{Nt}=0.7$, and $\varepsilon=20$, for FHF.

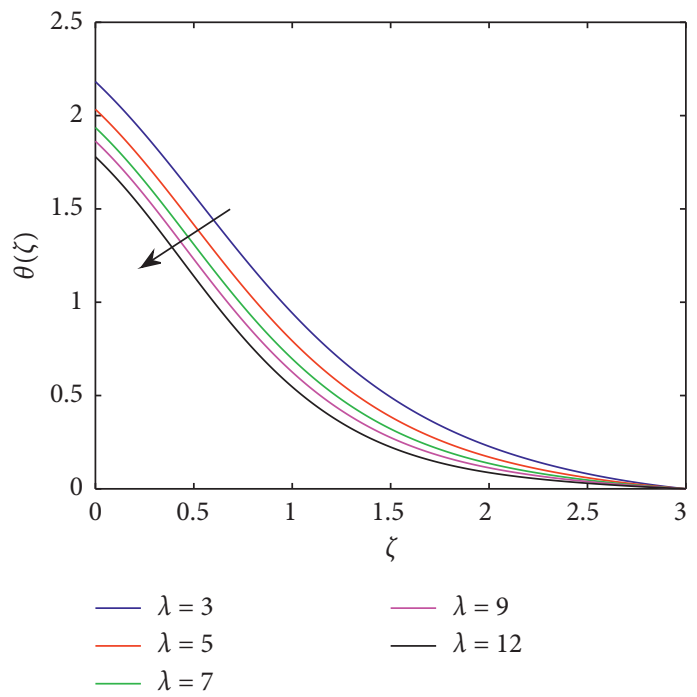

FIGURE 18: $\theta(\zeta)$ graph for different values of $\lambda$ when $\mathrm{Gm}=\mathrm{Gr}=\mathrm{bm}=\mathrm{bh}=\mathrm{Nt}=0.1$ and $\mathrm{bv}=P=1$, for FHF.

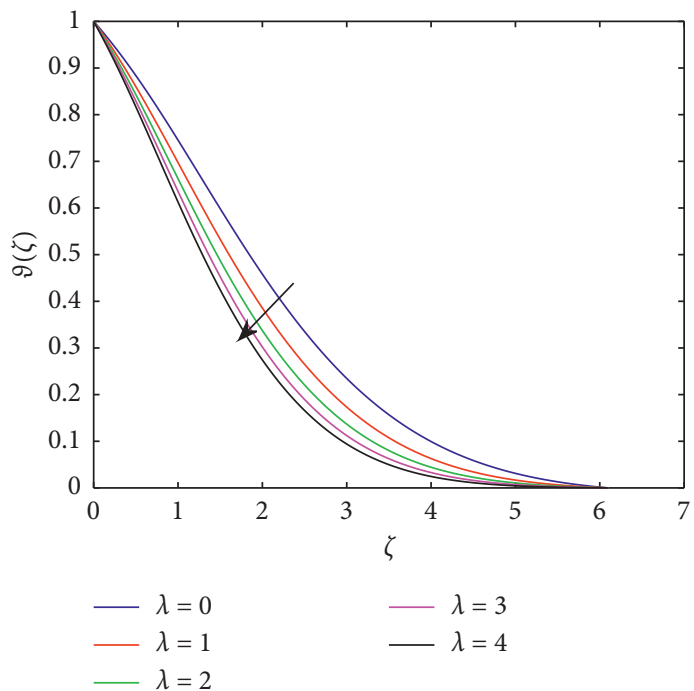

Figure 19: $\vartheta(\zeta)$ graph for different values of $\lambda$ when $\mathrm{Gr}=1.25, \mathrm{Gm}=0.1, \alpha=0.3$, and $\mathrm{Pr}=0.77$, for FST. 


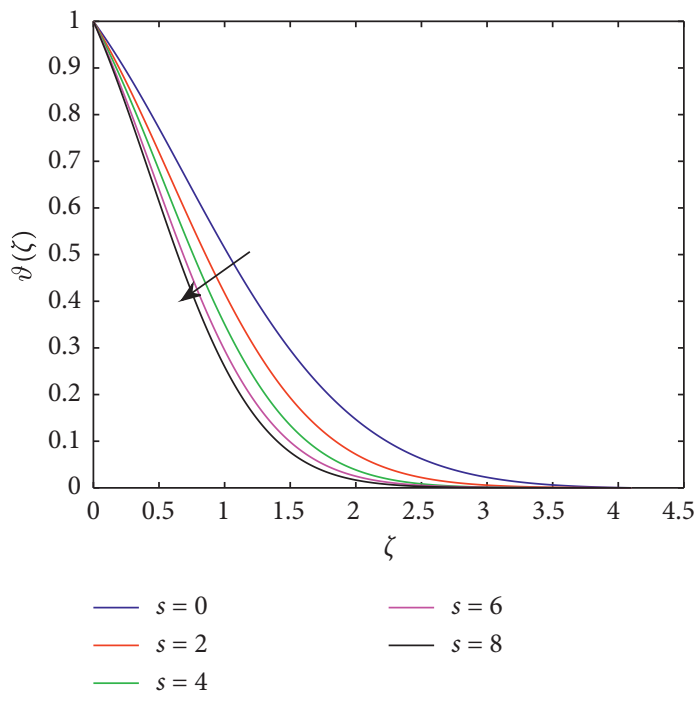

Figure 20: $\vartheta(\zeta)$ profile for different values of $s$ when $\mathrm{Gm}=\mathrm{Gr}=0.1, \mathrm{Nb}=1, \alpha=0$, and $\mathrm{Nt}=3$, for FST.

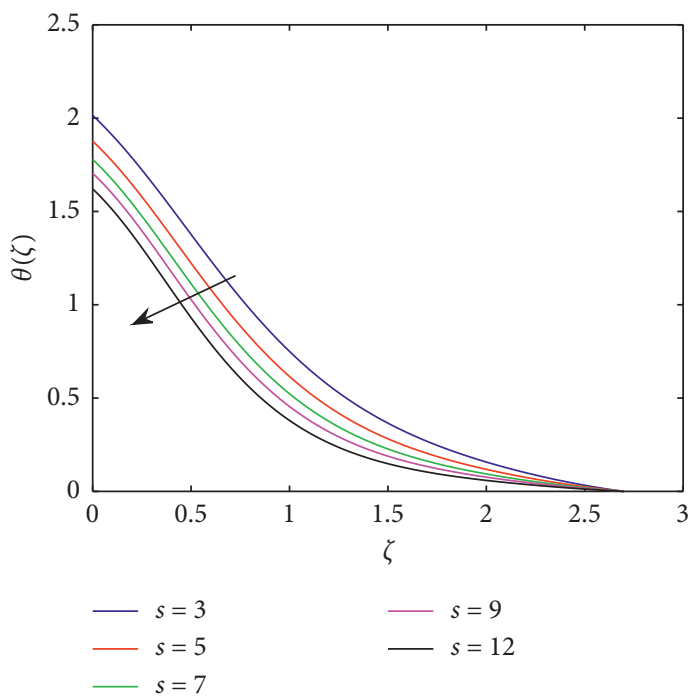

Figure 21: $\theta(\zeta)$ graph for different values of $s$ when $\mathrm{Gm}=0.72, \mathrm{Gr}=\mathrm{bm}=\mathrm{bh}=\mathrm{Nt}=0.1$, and $\mathrm{bv}=P=1$, for FHF.

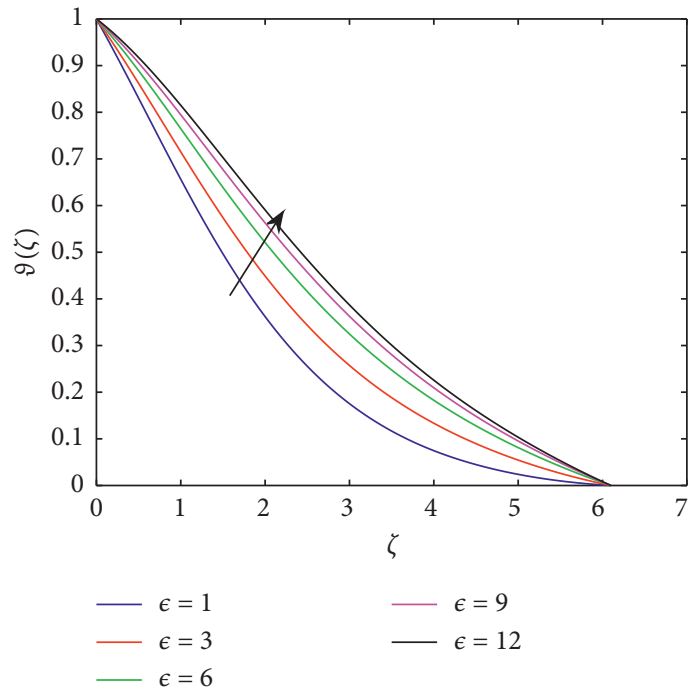

Figure 22: $\vartheta(\zeta)$ profile for different values of $\varepsilon$ when $\mathrm{Gr}=20, \mathrm{Gm}=\mathrm{Nb}=0.1, \lambda=\mathrm{bv}=\mathrm{bm}=\mathrm{bh}=0.5$, and $\mathrm{Nt}=3$, for $\mathrm{FST}$. 


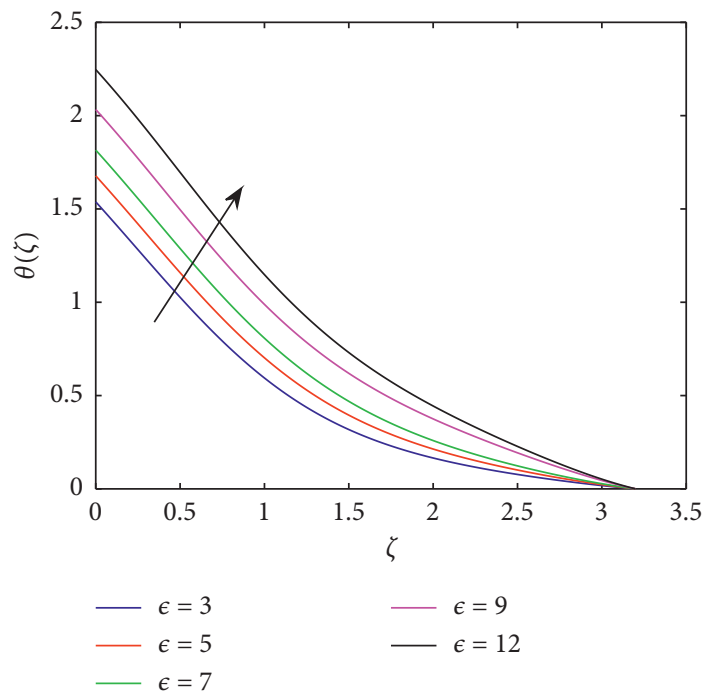

Figure 23: $\theta(\zeta)$ graph for different values of $\epsilon$ when $\mathrm{Gr}=\mathrm{bm}=\mathrm{bh}=\mathrm{Nt}=0.1, \lambda=\mathrm{bv}=\mathrm{Nb}=\mathrm{Gm}=0.5$, and $P=1$, for FHF.

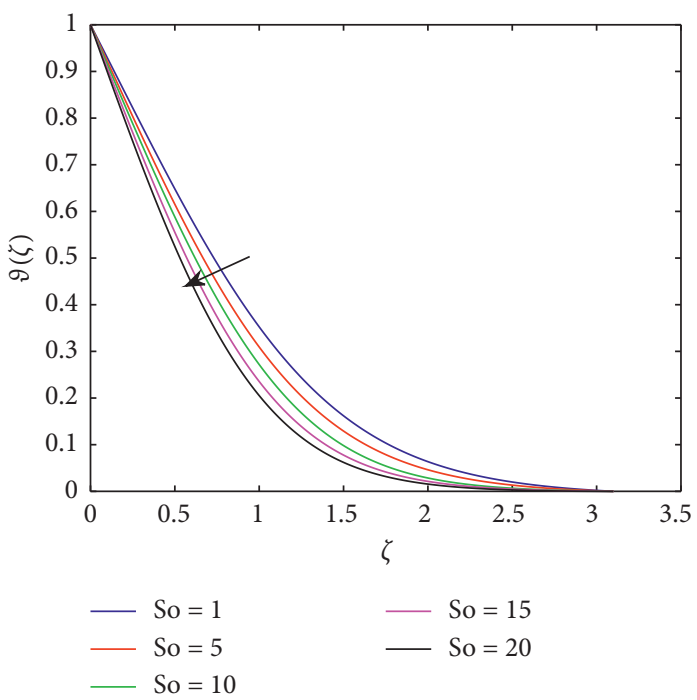

FIGURE 24: $\vartheta(\zeta)$ profile for different values of So when $\mathrm{Gm}=\mathrm{Gr}=\alpha=0.1, \lambda=\mathrm{bv}=\mathrm{bm}=\mathrm{bh}=0.5, m=1$, and $\mathrm{Nt}=0.4$, for FST.

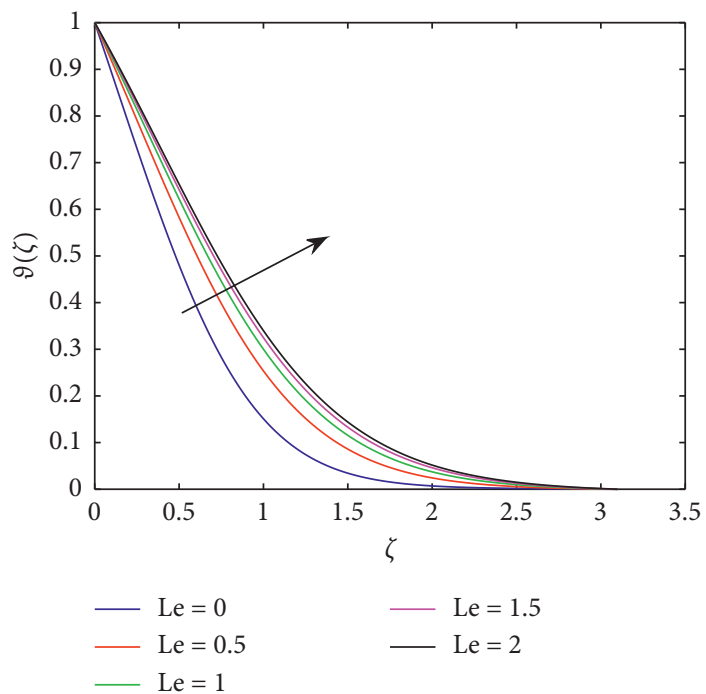

Figure 25: $\vartheta(0)$ graph for different values of Le when $\mathrm{Gm}=m=\mathrm{NB}=1, \mathrm{Gr}=\alpha=0.1, \lambda=\mathrm{bv}=\mathrm{bm}=\mathrm{bh}=0.5$, and $\mathrm{Nt}=0.4$, for FST. 


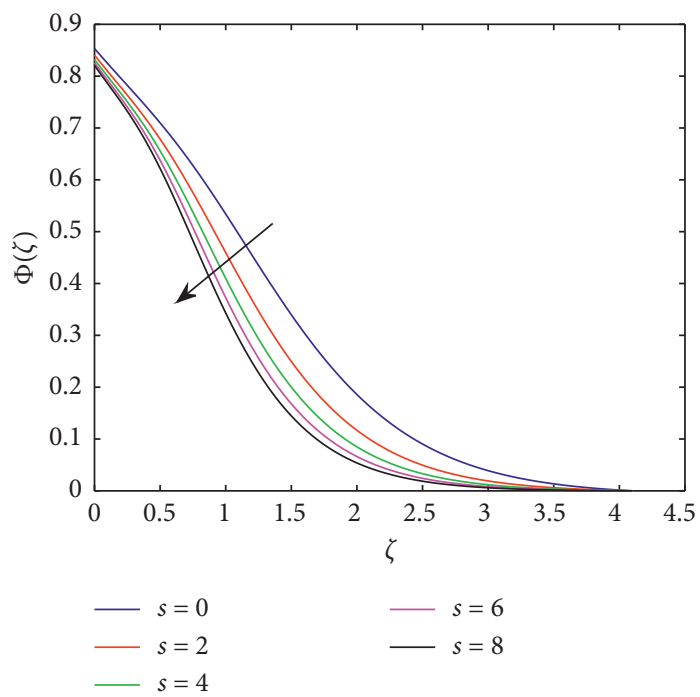

Figure 26: $\Phi(\zeta)$ graph for different values of $s$ when $\mathrm{Gm}=0.1, \mathrm{Gr}=1$, and $\mathrm{Nt}=0.4$.

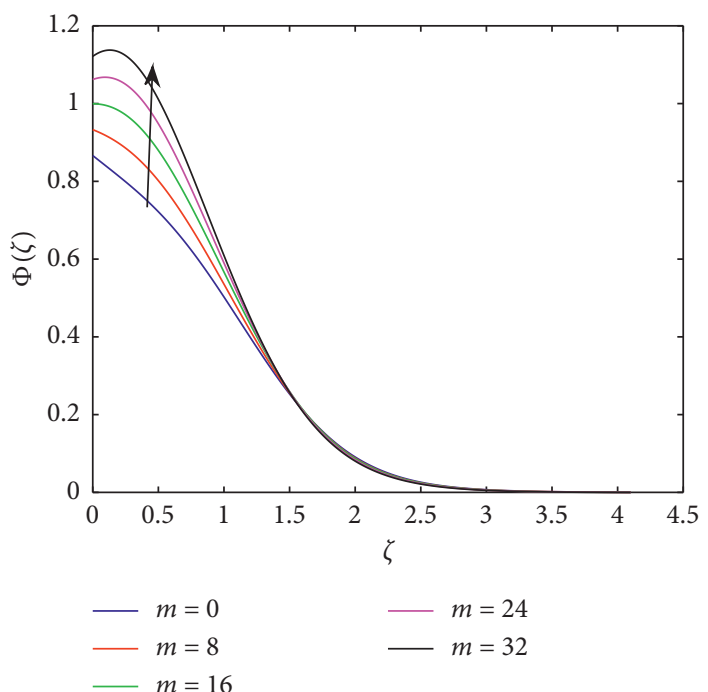

Figure 27: $\Phi(\zeta)$ profile for different values of $\mathrm{m}$ when $\mathrm{Gm}=1, \mathrm{Gr}=0.2, \mathrm{Pr}=\mathrm{Nb}=2,=\mathrm{bh}=0.5$, and $\mathrm{Nt}=0.1$.

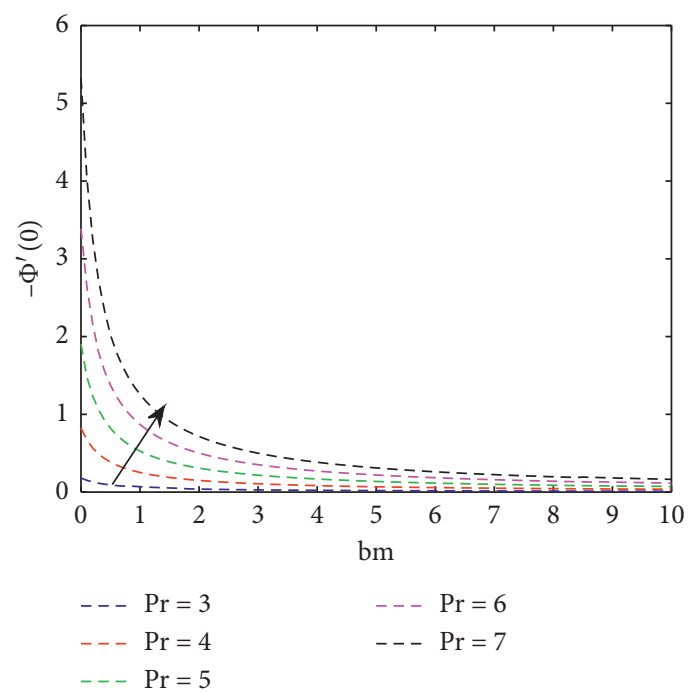

Figure 28: $-\Phi \prime(0)$ profile for different values of $\operatorname{Pr}$ when $\mathrm{Gr}=\mathrm{bh}=0.1, \lambda=\mathrm{bv}=\mathrm{bm}=\mathrm{bh}=0.5, \mathrm{Gm}=m=1$, and $\mathrm{Nt}=0.7$. 


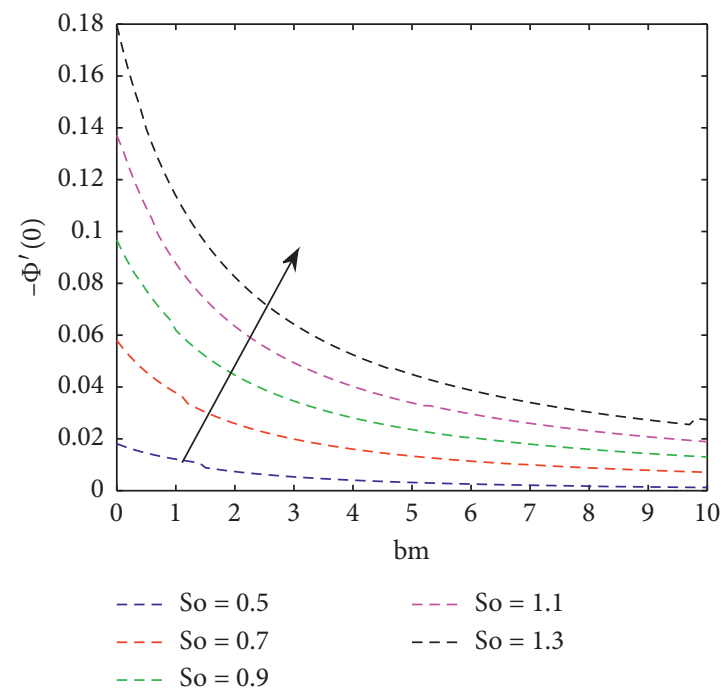

Figure 29: $-\Phi$ I (0) graph for different values of So when $\mathrm{Gm}=m=1, \mathrm{Gr}=\mathrm{bh}=0.1, \lambda=\mathrm{bv}=\mathrm{bm}=\mathrm{bh}=0.5$, and $\mathrm{Nt}=0.2$.

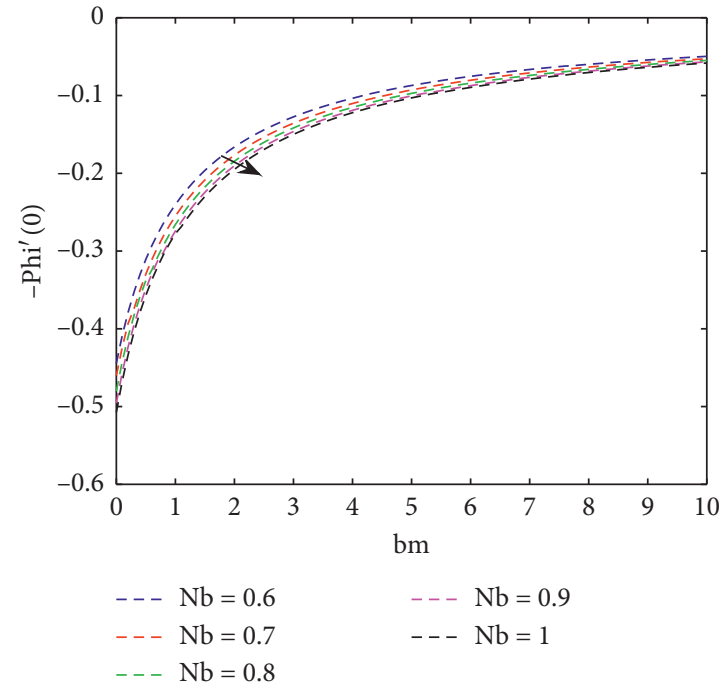

Figure 30: $-\Phi \prime(0)$ profile for different values of $\mathrm{Nb}$ when $\mathrm{Gm}=1, \mathrm{Gr}=0.1, \lambda=\mathrm{bv}=\mathrm{bm}=\mathrm{bh}=0.5$, and $\mathrm{Nt}=0.2$.

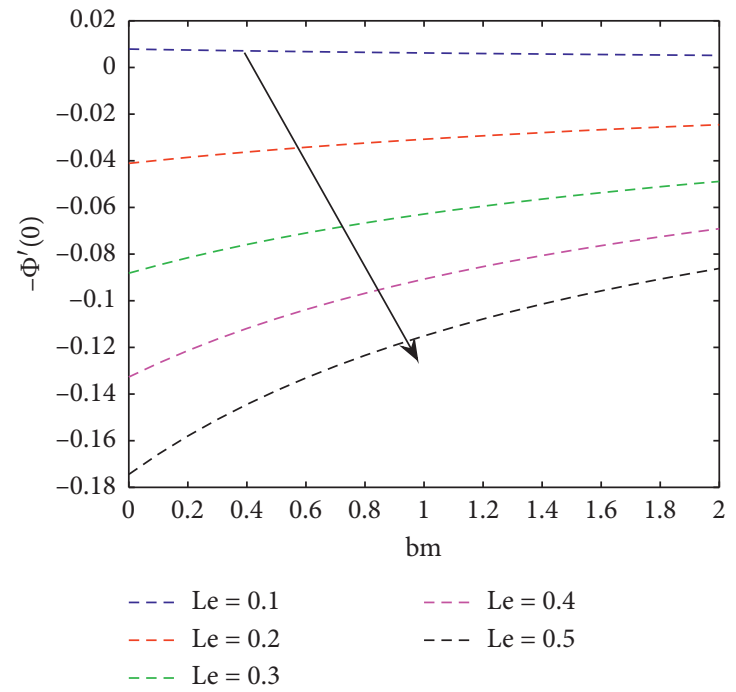

Figure 31: $-\Phi$ । $(\zeta)$ profile for different values of Le when $\mathrm{Gm}=\mathrm{Gr}=m=0.1 \mathrm{~s}=\alpha=0.1, \lambda=\mathrm{bv}=\mathrm{bm}=\mathrm{bh}=0.5$, and $\mathrm{Nt}=0.4$. 


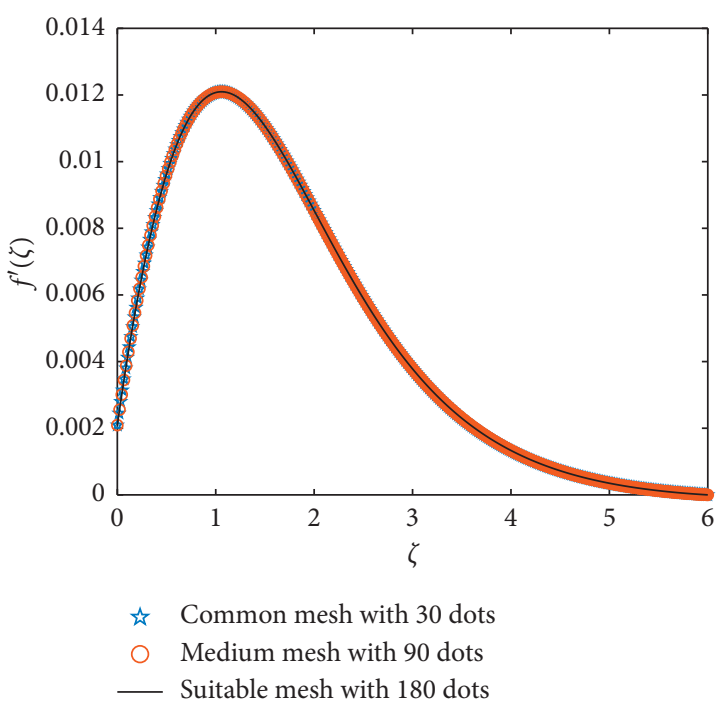

Figure 32: Grid-independence test for $f^{\prime}(\zeta)$ when $\mathrm{Gr}=13, m=1$, $\lambda=\mathrm{bv}=\mathrm{bm}=\mathrm{Nt}=n=\mathrm{So}=\mathrm{Le}=05, \mathrm{Gm}=\mathrm{bh}=\mathrm{bv}=s=\mathrm{Nb}=$ $\mathrm{Ma}=\alpha=0.1, n=09, \mathrm{Nb}=3$, and $\mathrm{Pr}=0.72$.

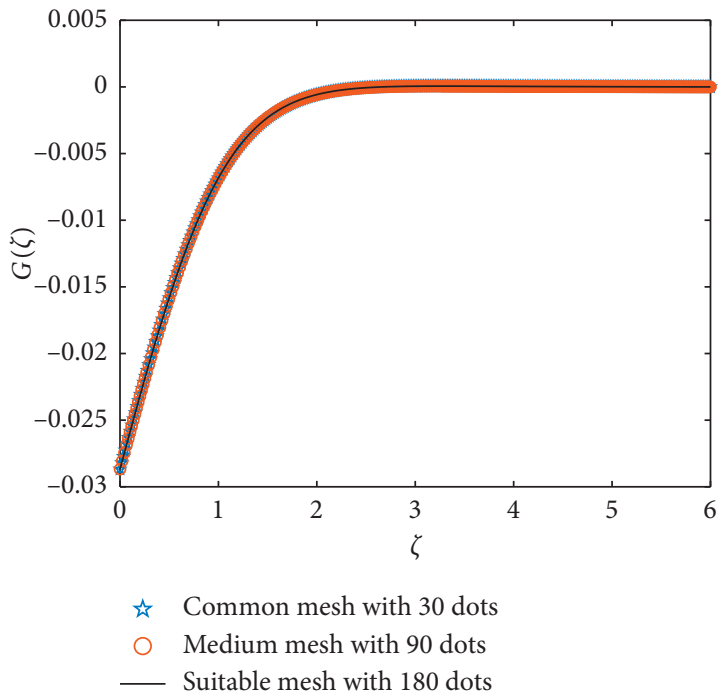

Figure 33: Grid-independence test for $G(\zeta)$ when $\mathrm{Gr}=$ $10, \mathrm{Gm}=m=1, \lambda=\mathrm{bv}=\mathrm{bm}=\mathrm{So}=\mathrm{Le}=05, \mathrm{bh}=s=\mathrm{NtMa}=\alpha=$ $0.1, n=09, \mathrm{Nb}=3$, and $\mathrm{Pr}=0.72$.

\section{Results and Discussion}

In this part, the effects of different governing physical parameters on dimensionless velocity, temperature, concentration, microrotation, skin friction, wall couple stress coefficients, and mass transfer coefficient have been discussed.

4.1. Velocity and Microrotation Profiles. The dimensionless velocity profile graphs of $f^{\prime}(\zeta)$, skin friction $f^{\prime \prime}(0)$, wall couple stresses $-G^{\prime}(0)$, and angular velocity profile $G(\zeta)$ for various magnitude of the microrotation slip parameter bh, velocity slip parameter bv, solutal and thermal nonlinear

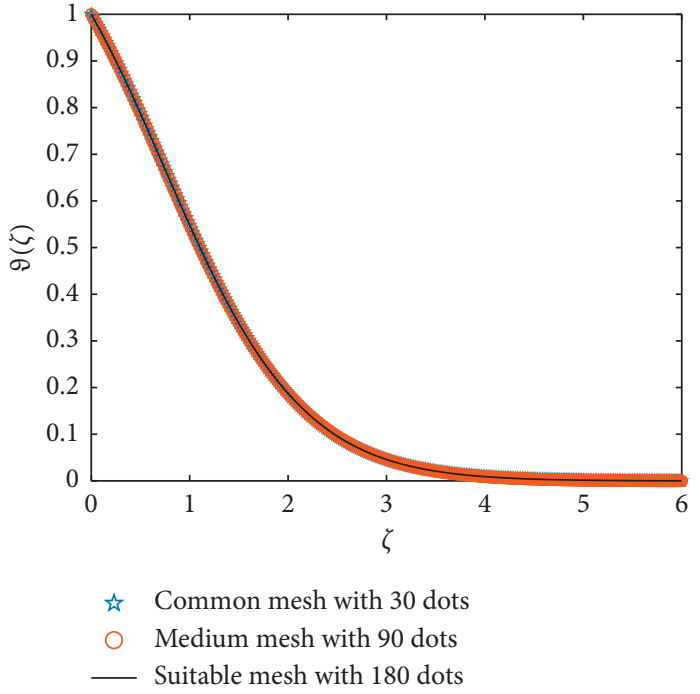

FIgURE 34: Grid-independence test for $\vartheta(\zeta)$ when $\mathrm{Gr}=$ $10, \mathrm{Gm}=m=1, \lambda=\mathrm{bv}=\mathrm{bm}=\mathrm{So}=\mathrm{Le}=05, \mathrm{bh}=s=\mathrm{NtMa}=\alpha=$ $0.1, n=09, \mathrm{Nb}=3$, and $\mathrm{Pr}=0.72$.

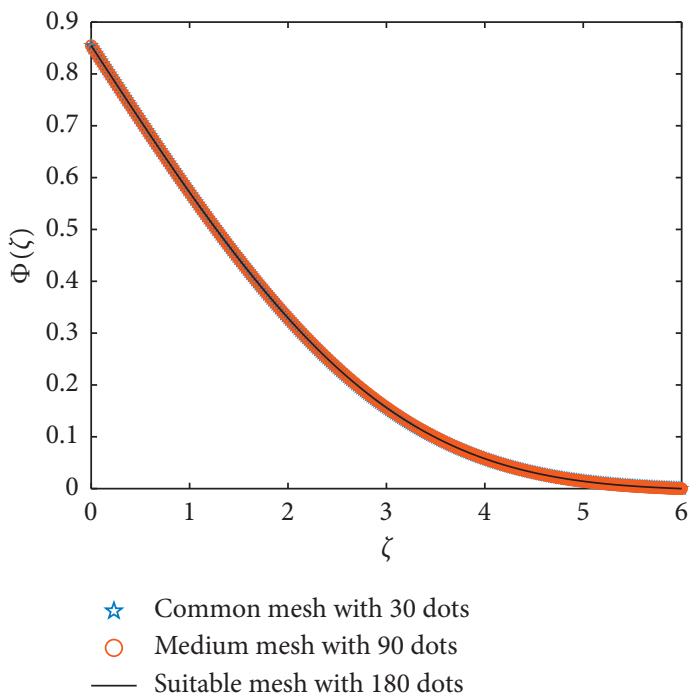

Figure 35: Grid-independence test for $\Phi(\zeta)$ when $\mathrm{Gr}=$ $10, \mathrm{Gm}=m=1, \lambda=\mathrm{bv}=\mathrm{bm}=\mathrm{So}=\mathrm{Le}=05, \mathrm{bh}=s=\mathrm{NtMa}=\alpha=$ $0.1, n=09, \mathrm{Nb}=3$, and $\operatorname{Pr}=0.72$.

TABle 1: Grid-independence test for velocity gradient $f^{\prime \prime}(\zeta)$ and angular velocity gradient $G^{\prime}(\zeta)$ when $n=\mathrm{Nt}=\mathrm{bm}=\mathrm{So}=$ $\mathrm{Le}=0.5, \mathrm{Pr}=0.7, \mathrm{Nb}=\mathrm{bv}=\mathrm{Gm}=\mathrm{bh}=s=0.1$, and $\mathrm{Gr}=13$.

\begin{tabular}{ccccccc}
\hline & \multicolumn{2}{c}{ Common mesh, } & \multicolumn{2}{c}{ Medium mesh, } & \multicolumn{2}{c}{ Suitable mesh, } \\
$\varepsilon$ & \multicolumn{2}{c}{30} & \multicolumn{2}{c}{90} & \multicolumn{2}{c}{180} \\
& $f^{\prime \prime}(\zeta)$ & $G^{\prime}(\zeta)$ & $f^{\prime \prime}(\zeta)$ & $G^{\prime}(\zeta)$ & $f^{\prime \prime}(\zeta)$ & $G^{\prime}(\zeta)$ \\
\hline 0 & 0.0319 & 0.0276 & 0.0319 & 0.027 & 0.0319 & 0.027 \\
1 & 0.0300 & 0.0250 & 0.0300 & 0.0250 & 0.0300 & 0.0250 \\
2 & 0.0287 & 0.0234 & 0.0278 & 0.0221 & 0.0278 & 0.0221 \\
3 & 0.0278 & 0.0221 & 0.0278 & 0.0221 & 0.0278 & 0.0221 \\
4 & 0.0269 & 0.0210 & 0.0269 & 0.0210 & 0.0269 & 0.0210 \\
5 & 0.0262 & 0.0201 & 0.0262 & 0.0201 & 0.0262 & 0.0201 \\
\hline
\end{tabular}


TABle 2: Grid-independence test for Nusselt number- $\vartheta^{\prime}(\zeta)$ and Sherwood number $-\Phi^{\prime}(\zeta) \quad$ when $n=0.9$, $\mathrm{Gm}=m=1, \mathrm{bv}=\mathrm{bm}=\mathrm{So}=\mathrm{Le}=0.5, \mathrm{Pr}=0.7, \mathrm{Nt}=\mathrm{bh}=s=\alpha=$ $\mathrm{Ma}=0.1, \mathrm{Nb}=3$, and $\mathrm{Gr}=10$.

\begin{tabular}{ccccccc}
\hline & \multicolumn{2}{c}{ Common mesh, } & \multicolumn{2}{c}{ Medium mesh, } & \multicolumn{2}{c}{ Suitable mesh, 180} \\
$\varepsilon$ & \multicolumn{2}{c}{30} & \multicolumn{2}{c}{90} & & \\
& $-\vartheta^{\prime}(\zeta)$ & $-\Phi^{\prime}(\zeta)$ & $-\vartheta^{\prime}(\zeta)$ & $-\Phi^{\prime}(\zeta)$ & $-\vartheta^{\prime}(\zeta)$ & $-G * \Phi^{\prime}(\zeta)$ \\
\hline 0 & 0.3697 & 0.3237 & 0.3697 & 0.3237 & 0.3697 & 0.3237 \\
1 & 0.3607 & 0.2955 & 0.3607 & 0.2955 & 0.3607 & 0.2955 \\
2 & 0.3497 & 0.2853 & 0.3497 & 0.2853 & 0.3497 & 0.2853 \\
3 & 0.3401 & 0.2777 & 0.3401 & 0.2777 & 0.3401 & 0.2777 \\
4 & 0.3315 & 0.2717 & 0.3315 & 0.2717 & 0.3315 & 0.2717 \\
5 & 0.3237 & 0.2665 & 0.3237 & 0.2665 & 0.3237 & 0.2665 \\
\hline
\end{tabular}

TABle 3: Comparison of Nusselt number $-\vartheta^{\prime}(0)$ when $n=0.5$ and $\operatorname{Pr}=0.7$, for different values of $\alpha$ and $\epsilon$, with previously published result.

\begin{tabular}{ccccc}
\hline & & $\begin{array}{c}\text { Present } \\
\text { result }\end{array}$ & $\begin{array}{c}\text { Nazar et al. } \\
{[15]}\end{array}$ & $\begin{array}{c}\text { Wubshet and Chaluma } \\
{[12]}\end{array}$ \\
$\alpha$ & $\varepsilon$ & $-\vartheta^{\prime}(0)$ & $-\vartheta^{\prime}(0)$ & $-\vartheta^{\prime}(0)$ \\
\hline 0.0 & 0.0 & 0.4576 & 0.4576 & 0.4577 \\
0.5 & & 0.4336 & 0.4336 & 0.4334 \\
1.0 & & 0.4165 & 0.4166 & 0.4166 \\
1.5 & & 0.4037 & 0.4035 & 0.4040 \\
2.0 & & 0.3931 & 0.3930 & 0.3932 \\
0.0 & 10 & 0.4561 & 0.4565 & 0.4560 \\
& 20 & 0.4535 & 0.4533 & 0.4534 \\
& 30 & 0.4481 & 0.4480 & 0.4486 \\
& 40 & 0.4406 & 0.4405 & 0.4406 \\
& 50 & 0.4309 & 0.4308 & 0.4309 \\
& 60 & 0.4189 & 0.4189 & 0.4188 \\
70 & 0.4048 & 0.4046 & \\
80 & 0.3878 & 0.3879 & \\
90 & 0.3687 & 0.3684 & \\
\hline
\end{tabular}

TABle 4: Comparison of Nusselt number $-\vartheta^{\prime}(0)$ when $n=0.5$ and $\operatorname{Pr}=7$, for different values of $\alpha$ and $\varepsilon$, with previously published result.

\begin{tabular}{lccc}
\hline$\alpha$ & $\varepsilon$ & $\begin{array}{c}\text { Present result } \\
-\vartheta^{\prime}(0)\end{array}$ & $\begin{array}{c}\text { Nazar et al. [15] } \\
-\vartheta^{\prime}(0)\end{array}$ \\
\hline 0.0 & 0.0 & 0.9598 & 0.9595 \\
0.5 & & 0.8904 & 0.8905 \\
1.0 & & 0.8443 & 0.8443 \\
1.5 & & 0.8096 & 0.8096 \\
2.0 & & 0.7809 & 0.7805 \\
\hline
\end{tabular}

convection $(s, \lambda)$, material parameters $\alpha$, dimensionless stream-wise coordinate $\varepsilon$, thermal and solutal Grashof numbers $(\mathrm{Gr}, \mathrm{Gm})$, nonisothermal parameters $(m, P)$, and magnetic parameter $\mathrm{Ma}$ are represented in Figures 2-15. Figures 2 and 6 , indicate that enhancing of $\alpha, n$, and bh reduces the density of the fluid which raise the opposition to rotate the fluid that cause increase the wall couple stresses $-G^{\prime}(0)$ and their boundary layer thickness.

Figure 3 shows that improving of $\mathrm{Gr}$ and bv decreases the kinematic viscosity of the fluid which raise the opposition to flow the fluid that cause decrease the skin friction coefficients $f^{\prime \prime}(0)$ and their boundary layer thickness. Figures 4, 5, and 7 reveal that an increase in $\mathrm{Gm}, s, \lambda$, and bv
TABLE 5: The computed values of skin friction coefficient $f^{\prime \prime}(0)$, wall couple stress $-G^{\prime}(0)$, Nusselt number $-\vartheta^{\prime}(0)$, and Sherwood number $-\Phi^{\prime}(0)$ when $\mathrm{Gr}=\mathrm{Nb}=\mathrm{Le}=\lambda=0.1, \mathrm{bv}=\mathrm{bh}=s=n=\mathrm{So}=0.5, \mathrm{Nt}=0.4$, $\alpha=1, \mathrm{Gm}=5$, and $\operatorname{Pr}=0.72$, for different values of $\mathrm{Ma}, P$, and $\epsilon$.

\begin{tabular}{lcccccc}
\hline $\mathrm{Ma}$ & $m$ & $\epsilon$ & $f^{\prime \prime}(0)$ & $-G^{\prime}(0)$ & $-\vartheta^{\prime}(0)$ & $-\Phi^{\prime}(0)$ \\
\hline 1 & 1 & 10 & 2.2812 & 2.0711 & 3.0357 & -5.5662 \\
1.5 & 1 & 10 & 2.3144 & 2.1159 & 3.0536 & -5.5928 \\
2 & 1 & 10 & 2.3480 & 2.1614 & 3.0712 & -5.6193 \\
1 & 0.1 & 10 & 2.2911 & -2.0841 & 3.0109 & \\
1 & 0.5 & 10 & 2.2867 & -2.0783 & 3.0221 & \\
1 & 1 & 10 & 2.2812 & -2.0711 & 3.0359 & \\
1 & 1.5 & 10 & 2.2757 & -2.0640 & 3.0497 & \\
1 & 1 & 1 & 2.4227 & & 3.0979 & -5.7126 \\
1 & 1 & 10 & 2.2812 & & 3.0359 & -5.5896 \\
1 & 1 & 20 & 2.1466 & & 2.8395 & -5.4360 \\
1 & 1 & 30 & 1.9795 & & 2.6535 & -5.2355 \\
1 & 1 & 40 & 1.8071 & & 2.5937 & -4.9782 \\
\hline
\end{tabular}

TABle 6: The computed values of skin friction coefficient $f^{\prime \prime}(0)$, wall couple stress $-G^{\prime}(0)$, and Sherwood number $-\Phi^{\prime}(0)$ when $\mathrm{Gr}=\mathrm{Nb}=\mathrm{Le}=\lambda=0.1, \mathrm{bv}=\mathrm{bh}=s=n=\mathrm{So}=0.5, \varepsilon=10 \mathrm{Nt}=0.4$, $\alpha=1, \mathrm{Gm}=5$, and $\operatorname{Pr}=0.72$, for different values of $\mathrm{Ma}$ and $P$.

\begin{tabular}{lcccc}
\hline $\mathrm{Ma}$ & $P$ & $f^{\prime \prime}(0)$ & $-G^{\prime}(0)$ & $-\Phi^{\prime}(0)$ \\
\hline 1 & 1 & 0.8882 & -0.4811 & -0.6793 \\
1.5 & 1 & 0.9226 & -0.5008 & -0.6812 \\
2 & 1 & 0.9464 & -0.5211 & -0.6829 \\
1 & 0.1 & 0.9598 & -0.5366 & -0.9159 \\
1 & 0.5 & 0.9342 & -0.5129 & -0.8157 \\
1 & 1 & 0.8992 & -0.4811 & -0.6793 \\
1 & 1.5 & 0.8601 & -0.4465 & -0.5282 \\
\hline
\end{tabular}

improves thermal and solutal volumetric expansion coefficients which improves the particle movement, and as a result, the skin friction coefficent $f^{\prime \prime}(0)$ is enhanced. Also, Figures 8-13 illustrate that increase of $m, \mathrm{Ma}, \lambda, s$, So, and $P$ boost the temperature difference between the boundary layer and ambient fluid to diffuse, which increases the velocity profile $f^{\prime}(\zeta)$ and their boundary layer thicknesses near the surface of the sphere and some distance far away from the wall (Figure 9). Moreover, when the values of $m=1$ and $m=120$, the graph of velocity profile $f^{\prime}(\zeta)$ is concave downwards near the points $(0.3648,0.1273)$ and $(0.2679$, $0.1874)$, respectively, as shown in Figure 8; as well as, when the values of $P=7$ and $P=35$, this profile is concave downwards near the points $(0.9555,0.03508)$ and (1.008, 0.03008), accordingly, as shown in Figure 13; while, for the value of $\lambda$, the velocity profile $f^{\prime}(\zeta)$ and its boundary layer thickness is decreasing in the interval of $\zeta[0.84,3]$, as shown in Figure 10. Furthermore, Figures 14 and 15 show that the increase of $\epsilon$ brings down the microrotation profile and the velocity profile near the wall of the sphere.

4.2. Temperature and Concentration Profiles. The importance of solutal jump parameter bm, Lewis and Prandtl numbers (Le and Pr), Brownian motion $\mathrm{Nb}$, thermophoresis $\mathrm{Nt}$, thermal nonlinear convection $\lambda$, solutal nonlinear convection parameters $s$, dimensionless stream-wise coordinate $\varepsilon$, Soret number So, nonisothermal parameters $(m, P)$, 
magnetic parameter Ma on temperature, concentration, and Sherwood numbers graphs are shown in Figures 16-33. Figures 16-21 indicate that increase of magnetic Ma, thermal nonlinear convection $\lambda$, and solutal nonlinear convection parameters $s$, enhances the density of the fluid which result in decline in both temperature distributions $\theta, \vartheta$ and their thermal boundary layer thickness. It is also observed that the temperature in both cases for FST and FHF reduces when the values of magnetic Ma, thermal nonlinear convection $\lambda$, and solutal nonlinear convection parameters $s$ improve at fixed values of $\zeta$. Similarly, the concentration distribution exhibits the reduction behavior for enlarge the values of solutal nonlinear convection parameters $s$, as shown in Figure 26. Moreover, Figure 24 demonstrates that boost of Soret number So enhances density of the fluid which associate through decrease in temperature and thermal boundary layer thicknesses; however, Figure 25 shows that enlarging of Lewis number Le enhances thermal conductivity and concentration difference between the boundary layer and ambient fluid to diffuse the temperature which is associated with the increase in temperature distribution as well as thermal boundary layer thickness.

Figures 22 and 23 show that improving of $\epsilon$ increases the thermal and solutal boundary layer of fluid which is associated with the enhance in both temperature distributions cases, i.e., in FST $\vartheta(\zeta)$ and FHF $\theta(\zeta)$. Similarly, Figure 27 reveals that boost of power index $m$ enlarge the solutal boundary layer thickness in the case of FST near the surface of the sphere.

Figures 28-31 demonstrate that upsurge of Prandtl number, Soret number, Lewis number, and Brownian motion parameter causes increase in the density of the fluid that results rise of the local Sherwood number. Figures 32-35 illustrate the grid-independence test. From these figures, it is observed that, for the number of points greater than suitable mesh number of points, the precision is not influenced but the set time is raised.

The presentation of grid-independence test is shown in Tables 1 and 2, to maintain the point of exactness known as grid convergence test. It started by the common mesh with 30 numbers of points. By enhancing number of points twice and triple, we get the medium mesh, 90 points of accuracy, and suitable mesh, 180 points of accuracy for velocity, angular, temperature, and concentration gradient values. For the number of points greater than suitable mesh number of points, the precision is not influenced but the set time is raised.

Tables 3 and 4 are drawn to compare the exactness of the method used in association with earlier reported data feasible in the literature studies which have been carried out. From Tables 1 and 2, it can be seen that the numerical magnitudes of the Nusselt number $-\vartheta^{\prime}(0)$, in the present study, for various values of $\chi$ and $\epsilon, n=0.5, \operatorname{Pr}=0.7$ and $\operatorname{Pr}=7$, are in an excellent agreement with the pervious results of published articles by Nazar et al. [15] and Wubshet and Chaluma [12]. The outcomes show that the numerical used in the information is truthful and highly accurate.

Tables 5 and 6 point out that upsurge in values of Ma causes enhancement in the skin friction coefficient $f^{\prime \prime}(0)$, wall couple stress coefficient $-G^{\prime}(0)$, Nusselt number in both cases $\left(-\vartheta^{\prime}(0),-\theta^{\prime}(0)\right)$, and Sherwood Number $-\Phi^{\prime}(0)$. This result happens due to improved density of the fluid which boosts the viscosity of it to flow, rotate, and diffuse that associate through enhancement of these physical quantities. However, as the magnitudes of $m$ and $\mathrm{P}$ increase, reduce the viscosity of the fluid flow which lowers opposition to flow and rotate the fluid that results in reduction of skin friction and wall couple stress coefficients. By similarl reasoning, Sherwood Number $-\Phi^{\prime}(0)$ of the fluid exhibits decreasing behavior as values of $P$ increase, but Nusselt number- $\vartheta^{\prime}(0)$ upsurge with $m$. However, increase in values of $\epsilon$ improves skin friction coefficient, Sherwood Number $-\Phi^{\prime}(0)$, and Nusselt number- $\vartheta^{\prime}(0)$.

\section{Conclusions}

This paper takes into account the effects of Soret, thermal and solutal nonlinear convection, nonisothermal, and magnetic parameters as well as manifold slip conditions on a micropolar nanofluid past a nonisothermal sphere, and the effects were analysed. The boundary layer equations of the flow problem are reduced into a duo of high-order nonlinear ordinary differential equations with the similarity transformation. The obtained differential equations are solved numerically with bvp4c from Matlab software. Numerical results are acquired for various main parameters of the flow problem. The results of governing parameters are reported by means of figures and tables. The main findings are

(1) An increase in the values of $m, \mathrm{Ma}, \lambda, s$, So, and $P$ allows to enhance the temperature difference between boundary layer and ambient fluid to diffuse, which increases the velocity profile $f^{\prime}(\zeta)$ and their boundary layer thicknesses near the surface of the sphere.

(2) The existence of Soret number So and nonisothermal parameter of FST $m$ agrees to reduce momentum kinematic viscosity which is associated with decrease in temperature distributions and their boundary layer thicknesses.

(3) An enhancement in the values of $\mathrm{Nt}$ and $\epsilon$ results in the boost of the temperature difference between the boundary layer and ambient fluid to diffuse the temperature which is associated with an increase in both temperature $(\vartheta(\zeta), \theta(\zeta))$ and concentration distributions.

(4) The tempereture distribution $\theta$ within the boundary layer can be enhanced by raising the values of nonisothermal parameter $\mathrm{P}$ which increase the density of the fluid; however, an increase in the value of $m$ reduces the surface temprature $\theta$.

(5) An improvement in magnitude of $\epsilon$ is made available to improve the microrotation profile, but it reduces the velocity profile within the boundary. This result happens due to improved density of the fluid

(6) The upsurge in values of Ma from 1 to 2 results in the improved density of the fluid which is associated 
with an enhancement in the skin friction coefficient $f^{\prime \prime}(0)$ by 2.9 percent, wall couple stress coefficient $-G^{\prime}(0)$ by 4.36 percent, Nusselt number $-9^{\prime}(0)$ by 1.16 percent, and Sherwood Number $-\Phi^{\prime}(0)$ by 0.94 percent.

(7) Skin friction and wall couple stress coefficients exhibit decreasing behavior by 0.67 and 10 percent and by 0.96 and 16 percent for increasing the magnitudes of $m$ and $P$ from 0.1 to 1.5. These effects are caused due to a decrease of viscosity of the fluid flow which lowers opposition to flow and to rotate the fluid in the boundary layer.

\section{Nomenclature}

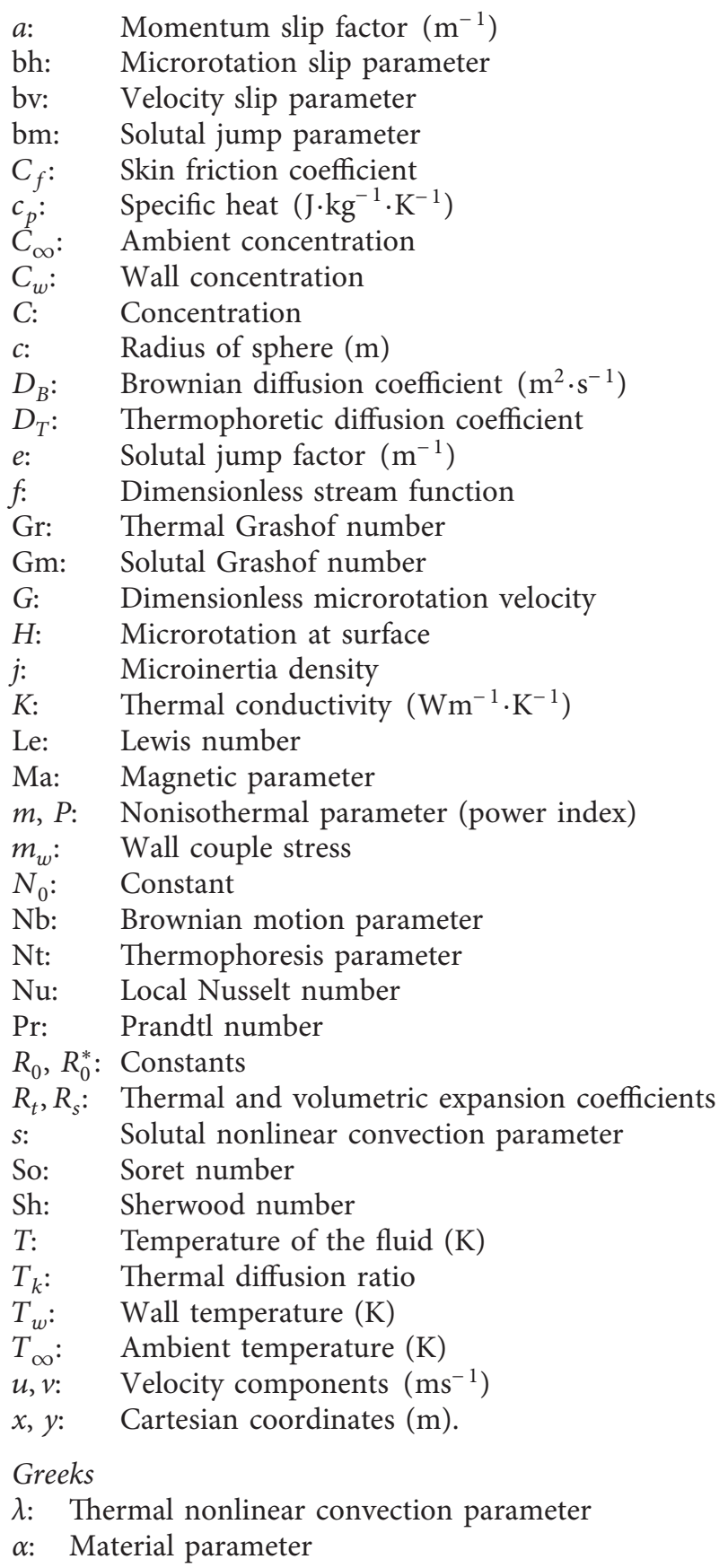

$\zeta$ : Dimensionless similarity variable

Э: Dimensionless temperature

$\Phi:$ Dimensionless Concentration

€: Dimensionless stream wise coordinate

$\mu$ : Coefficient of dynamic viscosity (Pas)

$\kappa$ : Vortex viscosity coefficient (Pas)

$v$ : Kinematic viscosity coefficient $\left(\mathrm{m}^{2} \cdot \mathrm{s}^{-1}\right)$

$\psi$ : Stream functions $\left(\mathrm{m}^{2} \cdot \mathrm{s}^{-1}\right)$

$\rho: \quad$ Fluid density $\left(\mathrm{k} \cdot \mathrm{gm}^{-3}\right)$

$\varphi$ : Spin-gradient viscosity $\left(\mathrm{m}^{2} \cdot \mathrm{s}^{-1}\right)$

$\tau_{w}:$ Wall shear stress (pa)

$\delta$ : Microrotation parameter.

Subscripts

$\infty$ : Conditions at the free stream

$w$ : Condition at the surface.

\section{Data Availability}

The data included in this paper are available without any restriction.

\section{Conflicts of Interest}

The authors declare that they have no conflicts of interest.

\section{References}

[1] K. V. Prasad, H. Vaidya, and K. Vajravelu, "MHD mixed convection heat transfer over a non-linear slender elastic sheet with variable fluid properties," Applied Mathematics and Nonlinear Sciences, vol. 2, no. 2, pp. 351-366, 2017.

[2] M. Kotouc, G. Bouchet, and J. A. M. Dusek, "Drag and flow reversal in mixed convection past a heated sphere," Physics of Fluid, vol. 21, no. 5, Article ID 054104, 2009.

[3] A. S. Rao, S. Sainath, P. Rajendra, and G. Ramu, "Mathematical modelling of hydromagnetic casson non-Newtonian nanofluid convection slip flow from an isothermal sphere," Nonlinear Engineering, vol. 8, no. 1, pp. 645-660, 2019.

[4] A. J. Chamkha and A. Al-Mudhaf, "Simultaneous heat and mass transfer from a permeable sphere at uniform heat and mass fluxes with magnetic field and radiation effects," $\mathrm{Nu}$ merical Heat Transfer, Part A: Applications, vol. 46, no. 2, pp. 181-198, 2004.

[5] C. S. Balla and K. Naikoti, "Finite element of analysis of magnetohydrodynamic transient free convection flow of nanofluid over a vertical cone with thermal radiation," Proceedings of the Institution of Mechanical Engineers, Part N: Journal of Nanomaterials, Nanoengineering and Nanosystems, vol. 230, no. 3, pp. 161-173, 2014.

[6] W. Lipinf, Y. Lu, and W. Jinjia, "Numerical study on the mixed convection heat transfer between a sphere particle and high pressure water in pseudocritical zone," Advances in Mechanical Engineering, vol. 5, Article ID 527182, 2013.

[7] S. A. Gaffar, K. Ur-Rehman, P. R. Reddy, V. R. Prasad, and B. M. H. Khan, "Powell-Eyring fluid flow towards an isothermal sphere in a non-darcy porous medium," Canadian Journal of Physics, vol. 97, no. 10, pp. 1039-1048, 2019.

[8] M. W. A. Dogbalous, M. Kinyanji, and M. Kimathi, "Effect of variable viscosity on unsteady natural convection hydromagnetic fluid flow over an isothermal sphere in a rotating system," International Journal of Advances in Applied Mathematics and Mechanics, vol. 7, no. 1, pp. 41-57, 2019. 
[9] H. A. Alzgool, H. T. Alkasasbeh, S. Abu-gharra, Z. Al-houri, and M. Z. Swalmeh, "Numerical solution of heat transfer in MHD mixed convection flow micropolar cassonfluid about solid sphere with radiation effect," International Journal of Engineering Research and Technology, vol. 12, no. 4, pp. 519-529, 2019.

[10] M. Z. Salleh, R. Nazar, and I. Pop, "Mixed convection boundary layer flow from a solid sphere with newtonian heating in a micropolarfluid," SRX Physics, vol. 2010, Article ID 736039, 9 pages, 2010.

[11] I. Wubshet and B. Shanker, "MHD boundary layer flow and heat transfer of a nanofluid over non- isothermal stretching sheet," Journal of Heat Transfer, vol. 136, pp. 051701-051709, 2014.

[12] I. Wubshet and Z. Chaluma, "Non-linear convection flow of micropolar nanofluid past an isothermal sphere," Journal of Physics Communications, vol. 3, no. 11, Article ID 115017, 2019.

[13] C. H. Amanulla, W. Abderrahim, B. Zoubair, M. S. Reddy, and N. Nagendra, "Numerical investigations on magnetic field modeling Carreau non-Newtonian fluid flow past an isothermal sphere," Journal of the Brazilian Society of Mechanical Science and Engineering, vol. 40, p. 462, 2018.

[14] L. F. Shampine, I. Glandwell, and S. Thomson, Solving ODEs with Matlab, Cambridge University Press, Cambridge, NY, USA, 2003.

[15] R. Nazar, N. Amin, T. Grosan, and I. Pop, "Free convection boundary layer on an isothermal sphere in a micropolar fluid," International Communications in Heat and Mass Transfer, vol. 29, no. 3, pp. 377-386, 2002. 